N. V. Trung

Nagoya Math. J.

Vol. 102 (1986), 1-49

\title{
TOWARD A THEORY OF GENERALIZED COHEN-MACAULAY MODULES
}

\author{
NGÔ VIÊT TRUNG
}

\section{Introduction}

Throughout this paper, $A$ denotes a noetherian local ring with maximal ideal $\mathfrak{m}$ and $M$ a finitely generated $A$-module with $d:=\operatorname{dim} M \geqq 1$.

Definition. $\quad M$ is called a generalized Cohen-Macaulay (abbr. C-M) module if

$$
l\left(H_{\mathrm{m}}^{i}(M)\right)<\infty
$$

for $i=0, \cdots, d-1$, where $l$ denotes the length and $H_{\mathrm{m}}^{i}(M)$ the $i$ th local cohomology module of $M$ with respect to $\mathfrak{m}$.

The notion of generalized C-M modules was introduced in [6]. It has its roots in a problem of D.A. Buchsbaum. Roughly speaking, this problem says that the difference

$$
I(\mathfrak{q} ; M):=l(M / \mathfrak{q} M)-e(\mathfrak{q} ; M)
$$

takes a constant value for all parameter ideals $\mathfrak{q}$ of $M$, where $e(\mathfrak{q} ; M)$ denotes the multiplicity of $M$ relative to $\mathfrak{q}$ [5]. In general, that is not true [30]. However, J. Stückrad and W. Vogel found that modules satisfying this problem enjoy many interesting properties which are similar to the ones of C-M modules and gave them the name Buchsbaum modules [22], [23]. That led in [6] to the study of modules $M$ with the property

$$
I(M):=\sup I(\mathfrak{q} ; M)<\infty
$$

where $\mathfrak{q}$ runs through all parameter ideals of $M$, and it turned out that they are just generalized C-M modules.

The class of generalized C-M module is rather large. For instance, most of the considered geometric local rings such as the ones of isolated singularities or of the vertices of affine cones over projective curves are

Received February 15, 1983. 
generalized C-M rings. So it would be of interest to establish a theory of generalized C-M modules.

Although the theory of Buchsbaum modules has been rapidly developed by works of S. Goto, P. Schenzel, J. Stückrad, W. Vogel (see the monograph [20], little is known about generalized C-M modules. Besides, it lacks something which connects both kinds of modules together. If one is acquainted enough with the few references on generalized C-M modules [6], [11], [18], one might have the notice that almost all properties of systems of parameters (abbr. s.o.p.'s) of Buchsbaum modules also hold for s.o.p.'s of generalized C-M modules which are contained in a large power of the maximal ideal. For instance, if $M$ is a generalized C-M module, there exists a positive integer $n$ such that

$$
I(\mathfrak{q} ; M)=I(M)
$$

for all parameter ideals $\mathfrak{q} \subseteq \mathfrak{m}^{n}$ of $M$. So, with regard to the origin of generalized C-M modules, one should try to explain the above phenomenon in studying s.o.p.'s $a_{1}, \cdots, a_{d}$ of $M$ with the property

$$
I\left(a_{1}, \cdots, a_{d} ; M\right)=I(M) .
$$

Such s.o.p.'s will be called standard.

The aim of this paper is to show that standard s.o.p.'s carry important informations on the structure of generalized C-M modules and that via this notion, one can derive the theory of Buchsbaum modules from the theory of generalized C-M modules.

Now we will describe the organization of this paper together with its main results. The paper is divided in 6 sections.

In Section 1 we recall some basic facts on generalized C-M modules which will be used in the sequence.

In Section 2 we establish the main properties of standard s.o.p.'s. First, we can define standard s.o.p.'s of $M$ very simply without assuming before that $M$ is a generalized C-M module. Consequently, we get a surprising criterion stating that $M$ is a generalized C-M module iff $M$ has a standard s.o.p. of this sense (Theorem 2.1). We can also characterize standard s.o.p.'s by means of local cohomology (Theorem 2.5). From this it follows that they are standard sequences in the sense of M. Brodmann [3], [4], and special $d$-sequences in the sense of C. Huneke [14], [15]. It should be mentioned that $d$-sequences enjoy many interesting properties 
and have been proved as very useful for different topics of Commutative Algebra, see [13], [27], [29].

In Section 3 we study ideals of $A$ which have the property that every s.o.p. of $M$ contained in them are standard. Such ideals will be called $M$-standard. $M$ being a Buchsbaum module just means that $m$ is $M$ standard. There are various characterizations of standard ideals (Propositions 3.1, 3.2, Theorems 3.4, 3.10) which not only recover all known characterizations of Buchsbaum modules but yield new ones too.

In Section 4 we use standard s.o.p.'s to study Hilbert-Samuel (abbr. $\mathrm{H}-\mathrm{S}$ ) functions. First, inspired of the characterization of $d$-sequences by means of their H-S functions [27], we give a polynomial bounding above the H-S function of an arbitrary s.o.p. of a generalized C-M module $M$ and show that they coincide iff this s.o.p. is standard (Theorem 4.1). Similarly, we can also estimate the H-S function of a submodule $N$ of $M$ of finite colength relative to an ideal a of $A$ with $l(M / \mathfrak{a} M)<\infty$ (Proposition 4.4). In particular, $M$ and $N$ will behave very well if $l(M / \mathfrak{a} N)$ attains some extreme value (Proposition 4.8). As a consequence, we are able to extend results of J. Sally [17] and S. Goto [8] on C-M and Buchsbaum rings with maximal embedding dimension for modules.

In Section 5 we show that if $\mathfrak{a}$ is a standard parameter ideal of $M$ or if $l\left(M / \mathfrak{a}^{2} M\right)$ attains some extreme value (the module-version of Buchsbaum rings with maximal embedding dimension), then the associated graded module

$$
G_{\mathfrak{a}}(M):=\bigoplus_{n=0}^{\infty} \mathfrak{a}^{n} M / \mathfrak{a}^{n+1} M
$$

is a homogeneous generalized C-M module and its local cohomology modules can be computed explicitly (Theorem 5.4 and Proposition 5.11). Moreover, we give a necessary and sufficient condition for an irrelevant ideal of $G_{\mathrm{q}}(A)$, where $q$ is a parameter ideal of $M$, to be $G_{\mathrm{q}}(M)$-standard (Theorem 5.7). Applying these results to Buchsbaum modules, we then get the main results of S. Goto in [8] and [9].

In Section 6 we shall first see that there is a close connection between $G_{a}(M)$ and the Rees module

$$
R_{\mathrm{a}}(M):=\bigoplus_{n=0}^{\infty} \mathfrak{a}^{n} M
$$

concerning the property of being a generalized C-M module (Proposition 6.1). If $\mathfrak{a}$ is a standard parameter ideal of $M$ or if $l\left(M / \mathfrak{a}^{2} M\right)$ attains some 
extreme value, then $\left[R_{\mathrm{a}}(M)\right]_{Q}$ is a generalized C-M module, where $Q$ denotes the maximal graded ideal of $R_{\mathrm{a}}(M)$, and its local cohomology modules can be computed explicitly (Theorem 6.2 and Proposition 6.5). As a consequence, we get necessary and sufficient conditions for $R_{a}(M)$ to be a Cohen-Macaulay module in these cases, generalizing recent results of S. Goto, Y. Shimoda, and P. Schenzel on this topic [7], [12], and [19].

It should be mentioned that special cases of Theorem 5.4, Proposition 5.11, and Theorem 6.2 are also obtained by M. Brodmann [4] and P. Schenzel (private communication).

Beside the notations introduced before, we shall use the following throughout this paper. Unless otherwise specified, $a_{1}, \cdots, a_{d}$ will be a s.o.p. of $M$. For convenience, we put

$$
\begin{aligned}
& \mathfrak{q}_{0}=0 \quad(\text { the zeroideal }) \\
& \mathfrak{q}_{i}=\left(a_{1}, \cdots, a_{i}\right) \quad(i=1, \cdots, d-1) \\
& \mathfrak{q}_{d}=\left(a_{1}, \cdots, a_{d}\right) .
\end{aligned}
$$

Moreover, we shall identify $H_{\mathrm{m}}^{0}(M)$ with the submodule $\bigcup_{n=1}^{\infty} 0_{M}: \mathfrak{m}^{n}$ of $M$ and denote by $\bar{M}$ the factor module $M / H_{\mathrm{m}}^{0}(M)$.

Acknowledgement. This paper was written while the author supported by a grant of the Matumae International Foundation was visiting the Department of Mathematics of Nagoya University. He would like to express his sincere thanks to both institutions and to Professor $\mathrm{H}$. Matsumura whose effort had made this visit possible. Compared with the first manuscript prepared before this visit, the present paper contains many improvements inspired by discussions with some Japanese mathematicians. The author is grateful to all of them, especially to S. Goto, S. Ikeda, and K. Yamagishi.

When the preparation of this paper was finished, the author learn that standard s.o.p.'s were also investigated by P. Schenzel in the preprints "Standard systems of parameters and their blowing-up rings" and "Extremal ideals and their form rings" but not in the complexity of the present paper. Especially, the theory of standard sequences has been further developed by Brodmann's papers "Local cohomology of certain Rees- and form-rings I, II" which contain, for example, results more general than Theorem 5.4 and Theorem 6.2.

Finally, the author would like to thank the referee for pointing out many mistakes of the manuscript. 


\section{§ 1. Basic facts}

First, generalized C-M modules may be characterized in different ways.

Lemma 1. [6, (3.3)]. The following conditions are equivalent:

(i) $M$ is a generalized $C-M$ module.

(ii) $I(M)<\infty$

(iii) There exists a s.o.p. $a_{1}, \cdots, a_{d}$ of $M$ such that

$$
\sup I\left(a_{1}^{n_{1}}, \cdots, a_{d}^{n_{d}} ; M\right)<\infty
$$

where $n_{1}, \cdots, n_{d}$ run through all positive integers.

(iv) There exists a positive integer $n$ such that

$$
\mathfrak{q}_{i-1} M: a_{i} \subseteq \mathfrak{q}_{i-1} M: \mathfrak{m}^{n}
$$

for every s.o.p. $a_{1}, \cdots, a_{d}$ of $M$ and $i=1, \cdots, d$.

The meaning of Lemma 1.1 (ii) has been already mentioned in the introduction of this paper. Lemma 1.1 (iii) is used to check whether a given module is a generalized C-M module or not. To explain the meaning of Lemma 1.1 (iv) we need the following notion of $[6,(2.3)]$ :

Definition. $M$ is called an $f$-module if every s.o.p. $a_{1}, \cdots, a_{d}$ of $M$ is a filter-regular $M$-sequence, i.e.

$$
\mathfrak{q}_{i-1} M: a_{i} \subseteq \bigcup_{n=1}^{\infty} \mathfrak{q}_{i-1} M: \mathfrak{m}^{n}
$$

for $i=1, \cdots, d$.

By Lemma 1.1 (iv), every generalized C-M module is an $f$-module. Of course, $f$-modules themselves have many interesting properties.

Lemma 1.2 [6, (2.5) and (2.11)]. The following conditions are equivalent:

(i) $M$ is an f-module.

(ii) Every s.o.p. $a_{1}, \cdots, a_{d}$ of $M$ is reducing, i.e.

$$
I(\mathfrak{q} ; M)=l\left(\mathfrak{q}_{d-1} M: a_{d} / \mathfrak{q}_{d-1} M\right) .
$$

(iii) Every s.o.p. $a_{1}, \cdots, a_{d}$ of $M$ is unmixed up to $\mathfrak{m}$, i.e. $\operatorname{dim} A / \mathfrak{p}=$ $d-i$ for all $\mathfrak{p} \in \operatorname{Ass}\left(M / \mathfrak{q}_{i} M\right) \backslash\{\mathfrak{m}\}$ and $i=0, \cdots, d-1$.

(iv) $M_{\mathfrak{p}}$ is a Cohen-Macaulay module with $\operatorname{dim} M_{\mathfrak{p}}=d-\operatorname{dim} A / \mathfrak{p}$ for all $\mathfrak{p} \in \operatorname{Supp}(M) \backslash\{\mathfrak{m}\}$. 
Remark 1.3. The notion of reducing s.o.p.'s was introduced by M. Auslander and D.A. Buchsbaum in [2, $\S 4]$. There they showed for every s.o.p. $a_{1}, \cdots, a_{d}$ of $M$ that

(1) $I(\mathfrak{q} ; M)=l\left(\mathfrak{q}_{d-1} M: a_{d} / \mathfrak{q}_{d-1} M\right)+\sum_{i=1}^{d-1} e\left(\mathfrak{q} ; \mathfrak{q}_{i-1} M: a_{i} / \mathfrak{q}_{i-1} M\right)$.

(2) $I(\mathfrak{q} ; M)=l\left(\mathfrak{q}_{d-1} M: a_{d} / \mathfrak{q}_{d-1} M\right)$ iff $a_{i} \notin \mathfrak{p}$ for all $\mathfrak{p} \in \operatorname{Ass}\left(M / \mathfrak{q}_{i-1} M\right)$ with $\operatorname{dim} A / \mathfrak{p} \geqq d-i$ and $i=1, \cdots, d$.

In most of practical situations, $f$-modules coincide with generalized C-M modules by the following result:

Lemma 1.4 [6, (3.8)]. Let $A$ be a factor of a $C-M$ ring. Then $M$ is an f-module iff $M$ is a generalized $C-M$ module (cf. also [32]).

In such situations, Lemma 1.2 (iv) provides a powerful criterion for generalized C-M modules. For example, it is easily seen from this criterion that the local rings of isolated singularities or of the vertices of affine cones over projective curves are always generalized C-M rings.

Now we will give some basic properties of generalized C-M modules.

Lemma 1.5 [6, (3.7)]. Let $M$ be a generalized $C-M$ module. Then

$$
I(M)=\sum_{i=0}^{d-1}\left(\begin{array}{c}
d-1 \\
i
\end{array}\right) l\left(H_{\mathrm{m}}^{i}(M)\right)
$$

Moreover, there exists a positive integer $n$ such that $I(\mathfrak{q} ; M)=I(M)$ for every parameter ideal $\mathfrak{q} \subseteq \mathfrak{m}^{n}$ of $M$.

Lemma 1.6. Let $M$ be a generalized $C$ - $M$ module. Then $\bar{M}$ is a generalized $C-M$ module with

(i) $H_{\mathrm{m}}^{0}(\bar{M})=0, H_{\mathrm{m}}^{i}(\bar{M}) \cong H_{\mathrm{m}}^{i}(M)$ for $i \geqq 1$.

(ii) $I(\bar{M})=I(M)-l\left(H_{\mathrm{m}}^{0}(M)\right)$.

Proof. (i) follows from the exact sequence

$$
0 \longrightarrow H_{\mathrm{m}}^{0}(M) \longrightarrow M \longrightarrow \bar{M} \longrightarrow 0 \text {. }
$$

(ii) is a consequence of Lemma 1.5 and (i).

Lemma 1.7. Let $M$ be a generalized $C$ - $M$ module with $d \geqq 2$. Let a be part of a s.o.p. of $M$. Then $M_{1}:=M / a M$ is a generalized $C-M$ module with

(i) $l\left(H_{\mathrm{m}}^{i}\left(M_{1}\right)\right) \leqq l\left(H_{\mathrm{m}}^{i}(M)\right)+l\left(H_{\mathrm{m}}^{i+1}(M)\right)$ for $i=0, \cdots, d-2$.

(ii) $I\left(M_{1}\right) \leqq I(M)$. 
Moreover, equality holds in (i) and (ii) iff $a H_{\mathrm{m}}^{i}(M)=0$ for all $i=0, \cdots$, $d-1$.

Proof. From the derived local cohomology sequence of the exact sequence

$$
0 \longrightarrow M / 0_{M}: a \stackrel{a}{\longrightarrow} M \longrightarrow M_{1} \longrightarrow 0
$$

we can easily deduce that

$$
l\left(H_{\mathrm{m}}^{i}\left(M_{1}\right)\right) \leqq l\left(H_{\mathrm{m}}^{i}(M)\right)+l\left(H_{\mathrm{m}}^{i+1}\left(M / 0_{M}: a\right)\right)
$$

for $i=0, \cdots, d-2$. Note that $0_{M}: a \subseteq H_{\mathrm{m}}^{\circ}(M)$ by Lemma 1.1 (iv). Then from the exact sequence

$$
0 \longrightarrow 0_{M}: a \longrightarrow M \longrightarrow M / 0_{M}: a \longrightarrow 0
$$

we get $H_{\mathrm{m}}^{i}\left(M / 0_{M}: a\right) \cong H_{\mathrm{m}}^{i}(M)$ for $i \geqq 1$. Hence (i) is obvious. Now, using Lemma 1.5 we have

$$
\begin{aligned}
I\left(M_{1}\right) & =\sum_{i=0}^{d-2}\left(\begin{array}{c}
d-2 \\
i
\end{array}\right) l\left(H_{\mathrm{m}}^{i}\left(M_{1}\right)\right) \\
& \leqq \sum_{i=0}^{d-2}\left(\begin{array}{c}
d-2 \\
i
\end{array}\right)\left[l\left(H_{\mathrm{m}}^{i}(M)\right)+l\left(H_{\mathrm{m}}^{i+1}(M)\right)\right] \\
& =\sum_{i=0}^{d-1}\left(\begin{array}{c}
d-1 \\
i
\end{array}\right) l\left(H_{\mathrm{m}}^{i}(M)\right)=I(M) .
\end{aligned}
$$

Clearly, equality holds above iff the sequence

$$
0 \longrightarrow H_{\mathrm{m}}^{i}(M) \longrightarrow H_{\mathrm{m}}^{i}\left(M_{1}\right) \longrightarrow H_{\mathrm{m}}^{i+1}\left(M / 0_{M}: a\right) \longrightarrow 0
$$

is exact for all $i=0, \cdots, d-2$. But that is the case iff $H_{\mathrm{m}}^{0}\left(M / 0_{M}: a\right)=0$, i.e. $a H_{\mathrm{m}}^{0}(M)=0$, and $a H_{\mathrm{m}}^{i}\left(M / 0_{M}: a\right)=a H_{\mathrm{m}}^{i}(M)=0$ for $i=1, \cdots, d-1$. So we have proved (ii) and the statement about equality.

\section{§2. Standard systems of parameters}

Definition. $a_{1}, \cdots, a_{d}$ is called a standard s.o.p. of $M$ if

$$
I\left(a_{1}^{2}, \cdots, a_{d}^{2} ; M\right)=I(\mathfrak{q} ; M) .
$$

This definition of standard s.o.p.'s is different from the one given in the introduction of this paper but leads to the same notion by the following result:

THEOREM 2.1. $a_{1}, \cdots, a_{d}$ is a standard s.o.p. of $M$ iff $M$ is a generalized $C-M$ module with $I(M)=I(\mathfrak{q} ; M)$. 
Properly speaking, Theorem 2.1 is a criterion for generalized C-M modules. It is rather surprising how a simple condition on a s.o.p. implies all the global properties of a generalized C-M module.

For the proof of Theorem 2.1 we shall need the following auxiliary result:

Lemma 2.2. Let $a_{1}, \cdots, a_{d}$ be an arbitrary s.o.p. of $M$. Then

$$
I\left(a_{1}^{n_{1}}, \cdots, a_{d}^{n_{d}} ; M\right) \leqq I\left(a_{1}^{m_{1}}, \cdots, a_{d}^{m_{d}} ; M\right)
$$

for all positive integers $n_{1} \leqq m_{1}, \cdots, n_{d} \leqq m_{d}$.

Proof. By induction we may assume that $n_{i}=m_{i}$ for $i<d$. Then

$$
\begin{aligned}
& l\left(\left(a_{1}^{n_{1}}, \cdots, a_{d-1}^{n_{d}-1}\right) M: a_{d}^{n_{d}} /\left(a_{1}^{n_{1}}, \cdots, a_{d-1}^{n_{d-1}}\right) M\right) \\
& \quad \leqq l\left(\left(a_{a}^{n_{1}}, \cdots, a_{d-1}^{n_{d}-1}\right) M: a_{d}^{m_{d}} /\left(a_{1}^{n_{1}}, \cdots, a_{d-1}^{n_{d}-1}\right) M\right) \\
& e\left(a_{1}^{n_{1}}, \cdots, a_{d}^{n_{d}} ;\left(a_{1}^{n_{1}}, \cdots, a_{i-1}^{n_{i}-1}\right) M: a_{i}^{n_{i}} /\left(a_{1}^{n_{1}}, \cdots, a_{i-1}^{n_{i}-1}\right) M\right) \\
& \quad=n_{d} e\left(a_{1}^{n_{1}}, \cdots, a_{d-1}^{d-1}, a_{d} ;\left(a_{1}^{n_{1}}, \cdots, a_{i-1}^{n_{i}-1}\right) M: a_{i}^{n_{i}} /\left(a_{1}^{n_{1}}, \cdots, a_{i-1}^{n_{i-1}}\right) M\right) \\
& \quad \leqq m_{d} e\left(a_{1}^{n_{1}}, \cdots, a_{d-1}^{n_{n}-1}, a_{d} ;\left(a_{1}^{n_{1}}, \cdots, a_{i-1}^{n_{i}-1}\right) M: a_{i}^{n_{i}} /\left(a_{1}^{n_{1}}, \cdots, a_{i-1}^{n_{i}-1}\right) M\right) \\
& \quad=e\left(a_{1}^{n_{1}}, \cdots, a_{d-1}^{n_{d}-1}, a_{d}^{m_{d}} ;\left(a_{1}^{n_{1}}, \cdots, a_{i-1}^{n_{i}-1}\right) M: a_{i}^{n_{i}} /\left(a_{1}^{n_{1}}, \cdots, a_{i-1}^{n_{i}-1}\right) M\right)
\end{aligned}
$$

for $i=1, \cdots, d-1$. Hence, applying Remark 1.3 (1) we get

$$
I\left(a_{1}^{n_{1}}, \cdots, a_{d}^{n_{d}} ; M\right) \leqq I\left(a_{1}^{n_{1}}, \cdots, a_{d-1}^{n_{d}-1}, a_{d}^{m_{d}} ; M\right) .
$$

Proof of Theorem 2.1. $\quad \Rightarrow$ ) By Lemma 1.1 (iii) and Lemma 1.5 we only need to show that

$$
I\left(a_{1}^{n_{1}}, \cdots, a_{d}^{n_{d}}, M\right)=I(\mathfrak{q} ; M)
$$

for all positive integers $n_{1}, \cdots, n_{d}$. First, using Lemma 2.2 and the definition of standard s.o.p.'s we get equality for $n_{1}, \cdots, n_{d} \in\{1,2\}$. If there exist positive integers $n_{1}, \cdots, n_{d}$ such that

$$
I\left(a_{1}^{n_{1}}, \cdots, a_{d}^{n_{d}} ; M\right) \neq I(\mathfrak{q} ; M),
$$

we must have $\max \left\{n_{1}, \cdots, n_{d}\right\}>2$. Without restriction we may assume that

$$
n_{d}=\max \left\{n_{1}, \cdots, n_{d}\right\}>2 .
$$

Then, by induction, we may further assume that

$$
I\left(a_{1}^{n_{1}}, \cdots, a_{d-1}^{n_{d}-1}, a_{d} ; M\right)=I\left(a_{1}^{n_{1}}, \cdots, a_{d-1}^{n_{d}-1}, a_{d}^{n_{d}-1} ; M\right)=I(\mathfrak{q} ; M) .
$$

Hence, looking at the proof of Lemma 2.2, we can conclude that 


$$
\begin{aligned}
& l\left(\left(a_{1}^{n_{1}}, \cdots, a_{d-1}^{n_{d}-1}\right) M: a_{d} /\left(a_{1}^{n_{1}}, \cdots, a_{d-1}^{n_{d-1}}\right) M\right) \\
& \quad=l\left(\left(a_{1}^{n_{1}}, \cdots, a_{d-1}^{n_{d}-1}\right) M: a_{d}^{n_{d-1}} /\left(a_{1}^{n_{1}}, \cdots, a_{d-1}^{n_{d}-1}\right) M\right), \\
& e\left(a_{1}^{n_{1}}, \cdots, a_{d-1}^{n_{d}-1}, a_{d} ;\left(a_{1}^{n_{1}}, \cdots, a_{i-1}^{n_{i-1}}\right) M: a_{i}^{n_{i}} /\left(a_{1}^{n_{1}}, \cdots, a_{i-1}^{n_{i-1}}\right) M\right)=0
\end{aligned}
$$

for $i=1, \cdots, d-1$. From the first equation we get

$$
\begin{aligned}
\left(a_{1}^{n_{1}}, \cdots, a_{d-1}^{n_{d}}\right) M: a_{d} & =\left(a_{1}^{n_{1}}, \cdots, a_{d-1}^{n_{d-1}}\right) M: a_{d}^{n_{d-1}} \\
& =\left(a_{1}^{n_{1}}, \cdots, a_{d-1}^{n_{d}-1}\right) M: a_{d}^{n_{d}},
\end{aligned}
$$

hence, applying Remark 1.3 (1),

$$
I\left(a_{1}^{n_{1}}, \cdots, a_{d}^{n_{d}} ; M\right)=I\left(a_{1}^{n_{1}}, \cdots, a_{d-1}^{n_{d}-1}, a_{d} ; M\right)=I(\mathfrak{q} ; M),
$$

a contradiction.

$(\Leftarrow)$ Since $I\left(a_{1}^{2}, \cdots, a_{d}^{2} ; M\right)=I(M)=I(\mathfrak{q} ; M)$, applying Lemma 2.2 we must have $I\left(a_{1}^{2}, \cdots, a_{d}^{2} ; M\right)=I(q ; M)$, as required.

For reduction process we shall need the following consequences of Theorem 2.1.

Corollary 2.3. $a_{1}, \cdots, a_{d}$ is a standard s.o.p. of $M$ iff $a_{1}, \cdots, a_{d}$ is a standard s.o.p. of $\bar{M}$ and $\mathfrak{q} M \cap H_{\mathrm{m}}^{0}(M)=0$.

Proof. Since $H_{\mathrm{m}}^{0}(M)$ is of finite length, we have $e(\mathfrak{q} ; \bar{M})=e(\mathfrak{q} ; M)$. Thus,

$$
\begin{aligned}
I(\mathfrak{q} ; \bar{M}) & =l\left(M / \mathfrak{q} M+H_{\mathfrak{m}}^{0}(M)\right)-e(\mathfrak{q} ; \bar{M}) \\
& =l(M / \mathfrak{q} M)-l\left(\mathfrak{q} M+H_{\mathrm{m}}^{0}(M) / \mathfrak{q} M\right)-e(\mathfrak{q} ; M) \\
& =I(\mathfrak{q} ; M)-l\left(H_{\mathrm{m}}^{0}(M) / \mathfrak{q} M \cap H_{\mathrm{m}}^{0}(M)\right) \\
& =I(\mathfrak{q} ; M)-l\left(H_{\mathrm{m}}^{0}(M)\right)+l\left(\mathfrak{q} M \cap H_{\mathrm{m}}^{0}(M)\right) .
\end{aligned}
$$

Now, using the relation $I(\bar{M})=I(M)-l\left(H_{\mathrm{m}}^{\circ}(M)\right)$ of Lemma 1.6 (ii), we easily get the statement.

Corollary 2.4. Let $M$ be a generalized $C$ - $M$ module with $d \geqq 2$. Then $a_{1}, \cdots, a_{d}$ is a standard s.o.p. of $M$ iff $a_{2}, \cdots, a_{d}$ is a standard s.o.p. of $M / a_{1} M$ and $I\left(M / a_{1} M\right)=I(M)$.

Proof. By Lemma 1.2 (ii) and Lemma 1.7, we have

$$
I\left(a_{2}, \cdots, a_{d} ; M / a_{1} M\right)=l\left(\mathfrak{q}_{d-1} M: a_{d} / M\right)=I(\mathfrak{q} ; M) .
$$

Hence the statement can be easily derived from Lemma 1.7 (ii).

Now we shall show that standard s.o.p.'s may be characterized by means of local cohomology (see Theorem 3.4 for further homological 
characterization).

ThEOREM 2.5. $a_{1}, \cdots, a_{d}$ is a standard s.o.p. of $M$ iff

$$
\mathfrak{q} H_{\mathrm{m}}^{i}\left(M / \mathfrak{q}_{j} M\right)=0
$$

for all non-negative integers $i, j$ with $i+j<d$.

Proof. Without restriction we may assume that $M$ is a generalized C-M module. If $d=1$, we have

$$
\begin{aligned}
& I\left(a_{1} ; M\right)=l\left(0_{M}: a_{1}\right) \\
& I\left(a_{1}^{2}: M\right)=l\left(0_{M}: a_{1}^{2}\right)
\end{aligned}
$$

by Lemma 1.2 (ii). Therefore, $a_{1}$ is a standard s.o.p. of $M$ iff $0_{M}: a_{1}=$ $0_{M}: a_{1}^{2}$ or, equivalently,

$$
0_{M}: a_{1}=\sum_{n=1}^{\infty} 0_{M}: a_{1}^{n}=\bigcup_{n=1}^{\infty} 0_{M}: \mathfrak{m}^{n}=H_{\mathrm{m}}^{0}(M) .
$$

So we have proved the case $d=1$. For $d>1$ we set $M_{1}=M / a_{1} M$.

If $a_{1}, \cdots, a_{d}$ is a standard s.o.p. of $M$, then $a_{1}, \cdots, a_{d}$ is also a standard s.o.p. of $M_{1}$ and $I\left(M_{1}\right)=I(M)$ by Corollary 2.4. By induction we have

$$
\mathfrak{q} H_{\mathrm{m}}^{i}\left(M / \mathfrak{q}_{j} M\right)=0
$$

for all $j \geqq 1, i+j<d$. Moreover, $a_{1} H_{\mathrm{m}}^{i}(M)=0$ for all $i=0, \cdots, d-1$ by Lemma 1.7. Hence, permuting $a_{1}, \cdots, a_{d}$ we also get $\mathfrak{q} H_{\mathrm{m}}^{i}(M)=0$ for $i=0, \cdots, d-1$.

Conversely, if

$$
\mathfrak{q} H_{\mathfrak{m}}^{i}\left(M / \mathfrak{q}_{j} M\right)=0
$$

for all $i+j<d$, then $a_{2}, \cdots, a_{d}$ is a standard s.o.p. of $M_{1}$ by induction and $I\left(M_{1}\right)=I(M)$ by Lemma 1.7. Hence $a_{1}, \cdots, a_{d}$ is a standard s.o.p. of $M$ by Corollary 2.4. The proof of Theorem 2.5 is now complete.

In [3], [4], M. Brodmann calls a sequence $b_{1}, \cdots, b_{r}$ of elements of $\mathfrak{m}$ an $\mathfrak{m}$-standard $M$-sequence if $b_{1}, \cdots, b_{r}$ is a filter-regular $M$-sequence and

$$
\left(b_{1}, \cdots, b_{r}\right) H_{\mathrm{m}}^{i}\left(M /\left(b_{1}, \cdots, b_{j}\right) M\right)=0
$$

for all non-negative integers $i, j$ with

$$
i+j<\max \left\{n ; l\left(H_{\mathrm{m}}^{t}(M)\right)<\infty \text { for } t<n\right\} .
$$


Hence, by Theorem 2.5, standard s.o.p.'s are m-standard sequences. That is why we choose the name "standard".

Theorem 2.5 has some interesting consequences.

Corollary 2.6. Let $a_{1}, \cdots, a_{d}$ be a standard s.o.p. of $M$. Then

(i ) $a_{1}, \cdots, a_{d}$ is a $d$-sequence of $M$, i.e.

$$
\mathfrak{q}_{i-1} M: a_{i} a_{j}=\mathfrak{q}_{i-1} M: a_{i}^{2}
$$

for $i=1, \cdots, d$ and $j>i$.

(ii) $a_{1}, \cdots, a_{d}$ is an absolutely superficial $M$-sequence, i.e.

$$
\left[\left(\mathfrak{q}^{n+1}, \mathfrak{q}_{i-1}\right) M: a_{i}\right] \cap \mathfrak{q} M=\left(\mathfrak{q}^{n}, \mathfrak{q}_{i-1}\right) M
$$

for all $n \geqq 0, i=1, \cdots, d$.

(iii) $\left(\mathfrak{q}_{i-1} M: a_{i}\right) \cap \mathfrak{q}\left(a_{i}, \cdots, a_{d}\right)^{n} M=\mathfrak{q}_{i-1}\left(a_{i}, \cdots, a_{d}\right)^{n} M$ for all $n \geqq 0$, $i=1, \cdots, d$.

(iv) $\mathfrak{q}_{i-1} M: a_{i}^{n}=\mathfrak{q}_{i-1} M: \mathfrak{q}^{m}$ for all $m, n>0, i=1, \cdots, d$.

(v) $\left(\mathfrak{q}^{n+1}, \mathfrak{q}_{i-1}\right) M: a_{i}=\mathfrak{q}^{n} M+\left(\mathfrak{q}_{i-1} M: a_{i}\right)$ for all $n>0, i=1, \cdots, d$.

Proof. By [27, Theorem 1.1, Corollary 1,2 (iii), and Acknowledgement], conditions (i) to (iv) are equivalent to each other and to the condition

$$
\mathfrak{q}_{i-1} M: a_{i}=\bigcup_{n=1}^{\infty} \mathfrak{q}_{i-1} M: \mathfrak{m}^{n}
$$

for all $i=1, \cdots, d$, and they imply (v). By Lemma 1.1 (iv), it is sufficient to show that

$$
\mathfrak{q}_{i-1} M: a_{i} \supseteq \bigcup_{n=1}^{\infty} \mathfrak{q}_{i-1} M: \mathfrak{m}^{n},
$$

which follows from the fact

$$
a_{i} H_{\mathrm{m}}^{0}\left(M / \mathfrak{q}_{i-1} M\right)=0
$$

of Theorem 2.5.

In particular, one can characterize standard s.o.p.'s by means of $d$ sequences as follows.

Proposition 2.7. $a_{1}, \cdots, a_{d}$ is a standard s.o.p. of $M$ iff by every permutation, $a_{1}^{n_{1}}, \cdots, a_{d}^{n_{d}}$ is a $d$-sequence of $M$ for all $n_{1}, \cdots, n_{d} \in\{1,2\}$.

Proof. $(\Rightarrow)$ By Theorem 2.1 and Lemma 2.2,

$$
I(M)=I(\mathfrak{q} ; M) \leqq I\left(a_{1}^{n_{1}}, \cdots, a_{d}^{n_{d}} ; M\right) \leqq I(M) .
$$


Therefore, $a_{1}^{n_{1}}, \cdots, a_{d}^{n_{d}}$ is also a standard s.o.p., hence a $d$-sequence of $M$ by Corollary 2.6 (i).

$(\Leftarrow)$ By [27, Theorem 1.1 (vii)] and Remark $1.3(2), a_{1}^{n_{1}}, \cdots, a_{d}^{n_{d}}$ is a reducing s.o.p. of $M$. Hence, using [27, Theorem 1.1 (vi)], we get

$$
\begin{aligned}
I\left(a_{\mathrm{i}}^{n}, \cdots, a_{d}^{n_{d}} ; M\right) & =l\left(\left(a_{1}^{n_{1}}, \cdots, a_{d-1}^{n_{d}-1}\right) M: a_{d}^{n_{d}} /\left(a_{1}^{n_{1}}, \cdots, a_{d-1}^{n_{d-1}}\right) M\right) \\
& =l\left(\left(a_{1}^{n_{1}}, \cdots, a_{d-1}^{n_{d}-1}\right) M: a_{d} /\left(a_{1}^{n_{1}}, \cdots, a_{d-1}^{n_{d}-1}\right) M\right) \\
& =I\left(a_{1}^{n_{1}}, \cdots, a_{d-1}^{n_{d}-1}, a_{d} ; M\right) .
\end{aligned}
$$

Now, permuting $a_{1}, \cdots, a_{d}$, we can easily show that

$$
I\left(a_{1}^{2}, \cdots, a_{d}^{2} ; M\right)=I(\mathfrak{q} ; M) \text {. }
$$

Remark 2.8. There are many criteria for $a_{1}, \cdots, a_{d}$ to be a $d$-sequence of $M$ [27, Theorem 1.1]. The simplest ones are the following:

(1) $\left(\mathfrak{q}_{i-1} M: a_{i}\right) \cap \mathfrak{q} M=\mathfrak{q}_{i-1} M$ for $i=1, \cdots, d$.

(2) $\mathfrak{q}_{i-1} M: a_{i}^{2} \subseteq \mathfrak{q}_{i-1} M: \mathfrak{q}$ for $i=1, \cdots, d$.

(3) $\mathfrak{q}_{i-1} M: a_{i}=\bigcup_{n=1}^{\infty} \mathfrak{q}_{i-1} M: \mathfrak{m}^{n}$ for $i=1, \cdots, d$.

The following result shows, together with Theorem 2.1, that some numerical invariants of $a_{1}, \cdots, a_{d}$ relative to $M$ will reach their maximal value iff $a_{1}, \cdots, a_{d}$ is a standard s.o.p. of $M$ (see also Theorem 4.1 and Corollary 4.2).

Proposition 2.9. Let $M$ be a generalized $C-M$ module. Then

$$
l\left(H_{\mathfrak{m}}^{i}\left(M / \mathfrak{q}_{j} M\right)\right) \leqq \sum_{t=i}^{i+j}(t-1) l\left(H_{\mathfrak{m}}^{t}(M)\right)
$$

for all non-negative integers $i, j$ with $i+j<d$. Equalities hold above by every permutation of $a_{1}, \cdots, a_{d}$ iff $a_{1}, \cdots, a_{d}$ is a standard s.o.p. of $M$.

Proof. For $j=0$ there is nothing to prove, For $j>0$ we have the following inequality

$$
l\left(H_{\mathrm{m}}^{i}\left(M / \mathfrak{q}_{j} M\right)\right) \leqq l\left(H_{\mathrm{m}}^{i}\left(M / \mathfrak{q}_{j-1} M\right)\right)+l\left(H_{\mathrm{m}}^{i+1}\left(M / \mathfrak{q}_{j-1} M\right)\right)
$$

by Lemma 1.7 (i). Applying this inequality successively, we then get the first statement. Moreover, by Lemma 1.7, equality holds above for a fixed $j<d$ and all $i=0, \cdots, d-j-1$ iff

$$
a_{j} H_{\mathfrak{m}}^{i}\left(M / \mathfrak{q}_{j-1} M\right)=0
$$

for all $i=0, \cdots, d-j$. Hence, using Theorem 2.5, we also get the second statement. 
We conclude this section by establishing, explicitly, the ubiquity of standard s.o.p.'s in a generalized C-M module, cf. Lemma 1.5.

Proposition 2.10. Let $M$ be a generalized $C$ - $M$ module. Let $\mathfrak{a}_{i}(M)$ denote the annihilator of $H_{\mathrm{m}}^{i}(M), i=0, \cdots, d-1$, and set

$$
\mathfrak{a}_{M}=\prod_{i=0}^{d-1} a_{i}(M)^{\left(\begin{array}{c}
d-1 \\
i
\end{array}\right)}
$$

Then every s.o.p. of $M$ contained in $\mathfrak{a}_{M}$ is standard.

Proof. Let $a_{1}, \cdots, a_{d}$ be a s.o.p. of $M$ contained in $\mathfrak{a}_{M}$. By Theorem 2.5, to show that $a_{1}, \cdots, a_{d}$ is a standard s.o.p. of $M$, it is sufficient to show that

$$
a_{i}\left(M / \mathfrak{q}_{j} M\right) \supseteq \mathfrak{a}_{M}
$$

for all non-negative integers $i, j$ with $i+j+d$. For $j=0$, that is immediate. For $j>0$, we have

$$
a_{i}\left(M / \mathfrak{q}_{j} M\right) \supseteq a_{i}\left(M / \mathfrak{q}_{j-1} M\right) a_{i+1}\left(M / \mathfrak{q}_{j-1} M\right)
$$

by the proof of Lemma 1.7 (i). Using this relation successively, we get

$$
a_{i}\left(M / \mathfrak{q}_{j} M\right) \supseteq \prod_{t=i}^{i+j} a_{t}(M)^{\left({ }_{t-i}^{j}\right)} \supseteq \mathfrak{a}_{M},
$$

because

$$
\left(\begin{array}{c}
j \\
t-i
\end{array}\right)=\left(\begin{array}{c}
j \\
i+j-t
\end{array}\right) \leqq\left(\begin{array}{c}
i+j \\
i+j-t
\end{array}\right)=\left(\begin{array}{c}
i+j \\
t
\end{array}\right) \leqq\left(\begin{array}{c}
d-1 \\
t
\end{array}\right)
$$

\section{§3. Standard ideals}

Throughout this section, $M$ will be a generalized C-M module and $a$ an ideal of $A$ with $l(M / \mathfrak{a} M)<\infty$.

Definition. $a$ is called an $M$-standard ideal if every s.o.p. of $M$ contained in $\mathfrak{a}$ is standard.

This notion extends the one introduced in [3], [4], where standard ideals are, roughly speaking, ideals generated by standard s.o.p.'s, cf. Corollary 3.3 below. The existence of standard ideals is guaranteed by Lemma 1.5 or, explicitly, by Proposition 2.10. In particular, $M$ being a Buchsbaum module just means that $m$ is $M$-standard.

First, we shall see that standard ideals may be also characterized by means of $d$-sequences and weak sequences. The latter ones were introduced in [27] as follows. 
Definition. A sequence of elements $b_{1}, \cdots, b_{r}$ of $A$ is called an $\mathfrak{a}$ weak $M$-sequence if

$$
\left(b_{1}, \cdots, b_{i-1}\right) M: b_{i} \subseteq\left(b_{1}, \cdots, b_{i-1}\right) M: \mathfrak{a}
$$

for all $i=1, \cdots, r$.

It should be mentioned that m-weak sequences are known as weak sequences and play an important role in the theory of Buchsbaum modules [22], [23].

Proposition 3.1. The following conditions are equivalent:

(i) a is $M$-standard.

(ii) Every s.o.p. of $M$ contained in a is an a-weak $M$-sequence.

(iii) Every s.o.p. of $M$ contained in a is a d-sequence of $M$.

Proof. (i) $\Rightarrow$ (ii). Let $a_{1}, \cdots, a_{d}$ be an arbitrary s.o.p. of $M$ contained in $a$. Let $S$ be a generating set for $a$ such that every $d$ element subset of $S \cup\left\{a_{1}, \cdots, a_{d}\right\}$ forms a s.o.p. of $M$, where the existence of such a set $S$ can be easily shown as in [21, Lemma 2] or [25, Lemma 3]. Then, by Corollary 2.6 (iv),

$$
\mathfrak{q}_{i-1} M: a_{i}=\bigcup_{n=1}^{\infty} \mathfrak{q}_{i-1} M: \mathfrak{m}^{n}=\bigcap_{a \in S} \mathfrak{q}_{i-1} M: a=\mathfrak{q}_{i-1} M: \mathfrak{a}
$$

for all $i=1, \cdots, d$. (ii) $\Rightarrow$ (iii) follows from [27, Proposition 2.2]. (iii) $\Rightarrow$ (i) follows from Proposition 2.7.

For practical uses, the following characterization of standard ideals is more convenient than Proposition 3.1 because it depends only on a finite system of elements.

In order to simplify our statement, we call a finite generating set $S$ for $\mathfrak{a}$ an $M$-base of $\mathfrak{a}$ if every $d$ element subset of $S$ forms a s.o.p. of $M$, see [21, Lemma 2] or [25, Lemma 3] for the existence of $M$-bases of a.

Proposition 3.2. a is $M$-standard iff one of the following conditions holds for all $d$ element subsets $\left\{a_{1}, \cdots, a_{d}\right\}$ of an M-base of $a$ :

(i) $a_{1}, \cdots, a_{d}$ is a standard s.o.p. of $M$.

(ii) $a_{1}^{n_{1}}, \cdots, a_{d}^{n_{d}}$ is an $\mathfrak{a}$-weak $M$-sequence for all $n_{1}, \cdots, n_{d} \in\{1,2\}$.

(iii) $a_{1}^{n_{1}}, \cdots, a_{d}^{n_{d}}$ is a d-sequence of $M$ for all $n_{1}, \cdots, n_{d} \in\{1,2\}$.

Proof. That a being $M$-standard implies (ii) follows from Proposition 3.1. (ii) $\Rightarrow$ (iii) is a consequence of $[27$, Proposition 2.2]. (iii) $\Rightarrow$ (i) follows from Proposition 2.7. Now suppose that (i) is satisfied. Then we have 
to show that every s.o.p. $b_{1}, \cdots, b_{a}$ of $M$ contained in $a$ is standard. If $d=1$, using Corollary 2.6 (iv) we have

$$
0_{M}: b_{1} \supseteq 0_{M}: a=\bigcap_{a \in S} 0_{M}: a=\bigcup_{n=1}^{\infty} 0_{M}: \mathfrak{m}^{n} \supseteq 0_{M}: b_{1} .
$$

Thus, $0_{M}: b_{1} \supseteq H_{\mathrm{m}}^{0}(M)$. Hence $b_{1}$ is a standard s.o.p. of $M$ by Theorem 2.5. If $d>1$, we can find a generating set $S^{\prime}$ for a such that $a_{1}, \cdots$, $a_{d-1}, b$ and $b_{1}, \cdots, b_{d-1}, b$ are s.o.p.'s of $M$ for all $b \in S^{\prime}$ and $\left\{a_{1}, \cdots, a_{d-1}\right\}$ $\subset S$ by the same method of [25, Lemma 3]. Using Corollary 2.6 (iv), we first have

$$
\mathfrak{q}_{d-1} M: b \supseteq \mathfrak{q}_{d-1} M: \mathfrak{a}=\bigcap_{a \in S} \mathfrak{q}_{d-1} M: a=\bigcup_{n=1}^{\infty} \mathfrak{q}_{d-1} M: \mathfrak{m}^{n} \supseteq \mathfrak{q}_{d-1} M: b
$$

and then

$$
\mathfrak{q}_{d-1} M: b=\bigcup_{n=1}^{\infty} \mathfrak{q}_{d-1} M: \mathfrak{m}^{n}=\mathfrak{q}_{d-1} M: a_{d} .
$$

Therefore, by virtue of Lemma 1.2 (ii) and Theorem 2.1,

$$
\begin{aligned}
I\left(a_{1}, \cdots, a_{d-1}, b ; M\right) & =l\left(\mathfrak{q}_{d-1} M: b / \mathfrak{q}_{-1} M\right)=l\left(\mathfrak{q}_{d-1} M: a_{d} / \mathfrak{q}_{d-1} M\right) \\
& =I(\mathfrak{q} ; M)=I(M),
\end{aligned}
$$

i.e. $a_{1}, \cdots, a_{d-1}, b$ is a standard s.o.p. of $M$. It follows by Corollary 2.4 that $a_{1}, \cdots, a_{d-1}$ is a standard s.o.p. of $M / b M$ and $I(M / b M)=I(M)$. Now, by induction, we may assume that $a$ is $M / b M$-standard. Then

$$
I\left(b_{1}, \cdots, b_{d-1}, b ; M\right)=I(M / b M)=I(M),
$$

i.e. $b_{1}, \cdots, b_{d-1}, b$ is a standard s.o.p. of $M$ too. Since the elements $b$ generate $a$, we can show, similarly as above, that

$$
I\left(b_{1}, \cdots, b_{d} ; M\right)=I(M) .
$$

The proof of Proposition 3.2 is now complete.

Proposition 3.2 immediately leads to the following consequence which justifies our notion of standard ideals from Brodmann's notion in [3], [4].

Corollary 3.3. Every ideal of $A$ generated by a standard s.o.p. of $M$ is $M$-standard.

Next, we shall show that standard ideals may be characterized by homological means, inspired from the so-called surjectivity criterion of Buchsbaum modules [21, Satz 1]. We point out that the proof for [21, 
Satz 1] could not be extended for our case.

First, we recall some facts about Koszul cohomology.

Let $K(S ; N)$ denote the Koszul complex of an arbitrary $A$-module $N$ with respect to a generating set $S$ of $a$. If $S=\left\{b_{1}, \cdots, b_{r}\right\}$, then $K(S ; N)$ may be viewed as the complex of the exterior products generated over $N$ by $r$ indeterminates $X_{1}, \cdots, X_{r}$ with the boundary map $X_{i} \rightarrow b_{i}, i=1$, $\cdots, r$. Let $H_{.}(S ; N)$ and $H^{\cdot}(S ; N)$ denote the homology and cohomology of $K(S ; N)$, respectively. Then

$$
H^{i}(S ; N) \cong H_{r-i}(S ; N)
$$

does not depend on the choice of $S$ and will be denoted by $H^{i}(a ; N)$, $i=0, \cdots, r$. It is well-known that

$$
H_{\mathrm{m}}^{i}(M)=\lim _{n} H^{i}\left(\mathfrak{a}^{n} ; M\right)
$$

for all $i=1, \cdots, d$.

Theorem 3.4. a is $M$-standard iff the natural homomorphism

$$
\varphi_{i}: H^{i}(\mathfrak{a} ; M) \longrightarrow H_{\mathrm{m}}^{i}(M)
$$

is surjective for $i=0, \cdots, d-1$.

Proof. $(\Rightarrow)$ Let $S$ be an $M$-base of a. Then

$$
0_{M}: \mathfrak{a}=\bigcap_{a \in S} 0_{M}: a=\bigcup_{n=1}^{\infty} 0_{M}: \mathfrak{m}^{n}
$$

by Corollary 2.6 (iv). Hence

$$
\varphi_{0}: H^{0}(\mathfrak{a} ; M)=0_{M}: \mathfrak{a} \longrightarrow H_{\mathfrak{m}}^{0}(M)=\bigcup_{n=1}^{\infty} 0_{M}: \mathfrak{m}^{n}
$$

is surjective. Thus, we may assume that $d>1$, and, we only need to show that $\varphi_{i}$ is surjective for $i=1, \cdots, d-1$. Note that $\bar{M}=M / H_{\mathrm{m}}^{0}(M)$. Then we have the commutative diagram

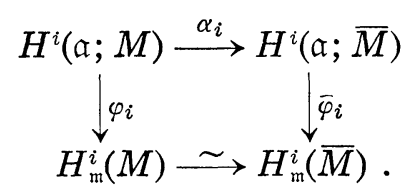

Consequently, $\varphi_{i}$ is surjective if $\alpha_{i}$ and $\bar{\varphi}_{i}$ are surjective.

Assume that $S=\left\{a_{1}, \cdots, a_{r}\right\}$. Then $\alpha_{i}$ is surjective if every $(r-i)$ th cycle $\bar{e}$ of $K(S ; \bar{M})$ is the natural image of some $(r-i)$ th cycle $e$ of 
$K(S ; M)$. Write

$$
\bar{e}=\sum \bar{m}_{(u)} X_{u_{1}} \cdots X_{u_{r-i}},
$$

$\bar{m}_{(u)} \in \bar{M}$, where $(u)$ runs through all sets $\left\{u_{1}, \cdots, u_{r-i}\right\}$ of integers between 1 and $r$. Let $m_{(u)}$ be elements of $M$ whose images in $\bar{M}$ are $\bar{m}_{(u)}$. Let $(v)=\left\{v_{1}, \cdots, v_{r-i-1}\right\}$ be a set of integers with $i \leqq v_{1}<\cdots<v_{r-i-1} \leqq r$ and $\left\{t_{1}, \cdots, t_{i+1}\right\}$ the complement of $(v)$ in $\{1, \cdots, r\}$. Put

$$
n_{(v)}=\sum_{j=1}^{i+1}(-1)^{\operatorname{sig}\left(t_{j}, v\right)} a_{t_{j}} m_{\left(t_{j}, v\right)} .
$$

Then $n_{(v)} \in H_{\mathrm{m}}^{0}(M)$ because $\bar{e}$ is a $(r-i)$ th cycle of $K(S ; \bar{M})$. Since

$$
H_{\mathrm{m}}^{0}(M) \cap\left(a_{t_{1}}, \cdots, a_{t_{i+1}}\right) M=0
$$

by Corollary 2.3, we must have $n_{(v)}=0$. That means

$$
e=\sum m_{(u)} X_{u_{1}} \cdots X_{u_{r-i}}
$$

is a $(r-i)$ th cycle of $K(S ; M)$, as required.

To show that $\bar{\varphi}_{i}$ is surjective we consider the exact sequence

$$
0 \longrightarrow M / 0_{M}: a_{1} \stackrel{a_{1}}{\longrightarrow} M \longrightarrow M / a_{1} M \longrightarrow 0 \text {. }
$$

Note that $0_{M}: a_{1}=\cup_{n=1}^{\infty} 0_{M}: \mathfrak{m}^{n}$ by Corollary 2.6 (iv). Then $M / 0_{M}: a_{1}=\bar{M}$ and we get the following commutative diagram

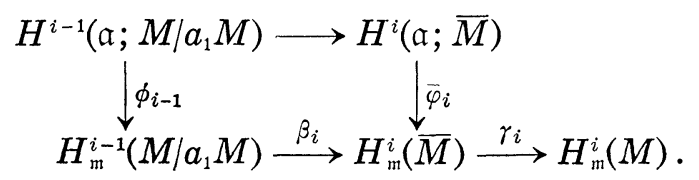

Since $\mathfrak{a}$ is $M / a_{1} M$-standard by Corollary 2.3 , by induction we may assume that $\phi_{i-1}$ is surjective. Further, since $a_{1} H_{\mathfrak{m}}^{i}(M)=0$ by Theorem 2.5, from the commutative diagram

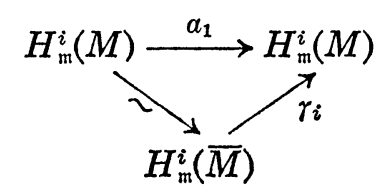

we can deduce that $\gamma_{i}$ is zero. Hence $\beta_{i}$ is surjective and so is $\bar{\varphi}_{i}$ too. So we have proved the necessary part.

$(\Rightarrow)$ We will show that every s.o.p. $a_{1}, \cdots, a_{b}$ of $M$ contained in a 
is a $d$-sequence of $M$, which then implies that $\mathfrak{a}$ is $M$-standard by Proposition 3.1. By Remark 2.8 (2) it suffices to show that

$$
\mathfrak{q}_{i-1} M: a_{i}^{2} \subseteq \mathfrak{q}_{i-1} M: \mathfrak{q}
$$

for $i=1, \cdots, d$. First, we have $a H_{\mathrm{m}}^{0}(M)=0$ because $\varphi_{0}$ is surjective. From this and Lemma 1.1 (iv) it follows that

$$
0_{M}: a_{1}^{2} \subseteq \bigcup_{n=1}^{\infty} 0_{M}: \mathfrak{m}^{n} \subseteq 0_{M}: \mathfrak{a} \subseteq 0_{M}: \mathfrak{q}
$$

Hence the case $i=1$ is immediate. For $i>1$ we consider the following exact sequences

$$
H^{i-1}\left(a_{1}^{n}, \cdots, a_{j}^{n} ; M\right) \stackrel{\gamma_{j}^{n}}{\longrightarrow} H^{i-1}\left(a_{1}^{n}, \cdots, a_{j-1}^{n} ; M\right) \stackrel{a_{j}^{n}}{\longrightarrow} H^{i-1}\left(a_{1}^{n}, \cdots, a_{j-1}^{n} ; M\right),
$$

$j=i+1, \cdots, d\left[2\right.$, Proposition 1.1]. Note that for $n$ large, $a_{1}^{n}, \cdots, a_{d}^{n}$ is a standard s.o.p. of $M$ by Lemma 1.5, hence a $d$-sequence by Corollary 2.6 (i). Then

$$
a_{j}^{n} H^{i-1}\left(a_{1}^{n}, \cdots, a_{j-1}^{n} ; M\right)=0
$$

by $[13, \S 5]$ (which was proved for rings but could be easily extended for modules). Hence $\gamma_{j}^{n}$ is surjective and so is

$$
\gamma_{d}^{n} \circ \cdots \circ \gamma_{i+1}^{n}: H^{i-1}\left(a_{1}^{n}, \cdots, a_{d}^{n} ; M\right) \longrightarrow H^{i-1}\left(a_{1}^{n}, \cdots, a_{i}^{n} ; M\right) .
$$

Thus, we have the following commutative diagram

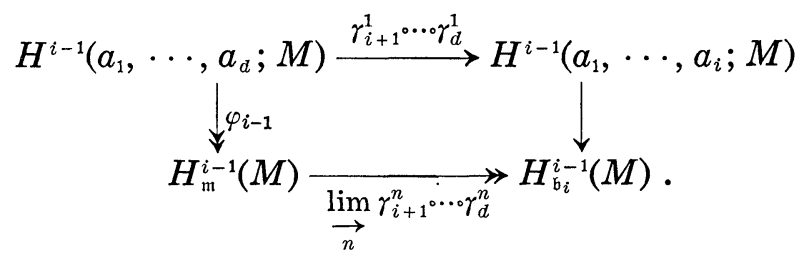

Now let $m_{i} \in q_{i-1} M: a_{i}^{2}$ arbitrary. Then

$$
a_{i}^{2} m_{i}=-\sum_{j=1}^{i-1} a_{j} m_{j}
$$

for some $m_{j} \in M$. Clearly, $m_{1}, \cdots, m_{i}$ define a cycle of $K\left(a_{1}, \cdots, a_{i-1}, a_{i}^{2} ; M\right)$

$$
e=\sum_{j=1}^{i} m_{j} X_{j}
$$

By virtue of the above diagram, we can find a cycle of $K\left(a_{1}, \cdots, a_{i-1}, a_{i}^{2} ; M\right)$ 


$$
f=\sum_{j=1}^{i} n_{j} X_{j}
$$

such that

(1) $f$ is the image of some $(d-i-1)$ th cycle of $K\left(a_{1}, \cdots, a_{d} ; M\right)$,

(2) The image of $e-f$ in $H_{q i}^{i-1}(M)$ is zero.

From (1) we can deduce that $a_{j} n_{i} \in \mathfrak{q}_{i-1} M$ for all $j=1, \cdots, d$, i.e. $n_{i} \in$ $\mathfrak{q}_{i-1} M: \mathfrak{q}$. From (2) we can deduce that there exist integers $n$ such that the image of $e-f$ in $K\left(a_{1}^{n}, \cdots, a_{i}^{n} ; M\right)$ is a boundary. From this it follows that

$$
\left(a_{1} \cdots a_{i-1}\right)^{n-1}\left(m_{i}-n_{i}\right) \in\left(a_{1}^{n}, \cdots, a_{i-1}^{n}\right) M .
$$

Note that by induction we have

$$
\mathfrak{q}_{j-1} M: a_{j}^{2} \subseteq \mathfrak{q}_{j-1} M: \mathfrak{q} \subseteq \mathfrak{q}_{j-1} M: \mathfrak{q}_{i-1} \subseteq \mathfrak{q}_{j-1} M: a_{j}^{2}
$$

and hence $\mathfrak{q}_{j-1} M: a_{j}^{2}=\mathfrak{q}_{j-1} M: \mathfrak{q}_{i-1}$ for $j=1, \cdots, i-1$. Then $a_{1}, \cdots, a_{i-1}$ is a $d$-sequence of $M$ by [27, Theorem $1.1(\mathrm{v})]$. So we may assume that every subsystem of parameters of $M$ of $i-1$ elements contained in $\mathfrak{q}$ is a $d$-sequence of $M$ and therefore a q-weak $M$-sequence by [27, Proposition 2.3]. Now, by virtue of the following Lemma 3.5, we have

$$
m_{i}-n_{i} \in\left(a_{1}^{n}, \cdots, a_{i-1}^{n}\right) M:\left(a_{1} \cdots a_{i-1}\right)^{n-1} \subseteq \mathfrak{q}_{i-1} M: \mathfrak{q} .
$$

Hence, $m_{i} \in \mathfrak{q}_{i-1} M: \mathfrak{q}$, as required. The proof of Theorem 3.4 is now complete.

The following auxiliary result is of independent interest because it establishes the monomial property of certain kind of sequences of elements of $A$.

Lemma 3.5 (cf. [9, Theorem 4.7]). Suppose that $b_{1}, \cdots, b_{r}$ is a system of elements in some ideal $\mathfrak{b}$ of $A$ such that by every permutation, $b_{1}^{n_{1}}, \cdots, b_{r}^{n_{r}}$ is a $\mathfrak{b}$-weak $M$-sequence for all positive integers $n_{1}, \cdots, n_{r}$. Then

$$
\left(b_{1}^{n}, \cdots, b_{r}^{n}\right) M ;\left(b_{1} \cdots b_{r}\right)^{n-1}=\left(b_{1}, \cdots, b_{r}\right) M+\sum_{j=1}^{r}\left(b_{1}, \cdots, b_{j}, \cdots, b_{r}\right) M: \mathfrak{b}
$$

for all $n \geqq 2$.

Proof. It is sufficient to show that

$$
\left(b_{1}^{n}, \cdots, b_{r}^{n}\right) M:\left(b_{1} \cdots b_{r}\right)^{n-1} \subseteq\left(b_{1}, \cdots, b_{r}\right) M+\sum_{j=1}^{r}\left(b_{1}, \cdots, \not b, \cdots, b_{r}\right) M: \mathfrak{b} .
$$

The case $r=1$ is immediate. For $r>1$ let $m$ be an arbitrary element of $\left(b_{1}^{n}, \cdots, b_{r}^{n}\right) M:\left(b_{1} \cdots b_{r}\right)^{n-1}$. Then, by induction, 


$$
b_{1}^{n-1} m \in\left(b_{1}^{n}, b_{2}, \cdots, b_{r}\right) M+\sum_{j=2}^{r}\left(b_{1}^{n}, b_{2}, \cdots, b_{j}, \cdots, b_{r}\right) M: \mathfrak{b} .
$$

Thus, there exist elements $m_{j} \in\left(b_{1}^{n}, b_{2}, \cdots, b_{j}, \cdots, b_{r}\right) M: \mathfrak{b}$ such that

$$
b_{1}^{n-1} m=\sum_{j=2}^{r} m_{j} \quad \text { modulo }\left(b_{2}, \cdots, b_{r}\right) M .
$$

Now we want to show that

$$
b_{1} m_{j} \in b_{1}^{n}\left[\left(b_{1}, \cdots, b_{j}, \cdots, b_{r}\right) M: \mathfrak{b}\right]+\left(b_{2}, \cdots, b_{r}\right) M .
$$

First, we can find elements $n_{j}, n_{j}^{\prime} \in M$ such that

$$
\begin{aligned}
& b_{1} m_{j}=b_{1}^{n} n_{j} \\
& b_{j} m_{j}=b_{1}^{n} n_{j}^{\prime}
\end{aligned}
$$

modulo $\left(b_{2}, \cdots, b_{j}, \cdots, b_{r}\right) M$. From this it follows that

$$
\begin{aligned}
b_{j} n_{j}-b_{1} n_{j}^{\prime} & \in\left(b_{2}, \cdots, b_{j}, \cdots, b_{r}\right) M: b_{1}^{n} \\
& \subseteq\left(b_{2}, \cdots, b_{j}, \cdots, b_{r}\right) M: \mathfrak{b} \subseteq\left(b_{2}, \cdots, b_{j}, \cdots, b_{r}\right) M: b_{j} .
\end{aligned}
$$

Hence

$$
n_{j} \in\left(b_{1}, \cdots, b_{j}, \cdots, b_{r}\right) M: b_{j}^{2} \subseteq\left(b_{1}, \cdots, b_{j}, \cdots, b_{r}\right) M: \mathfrak{b} .
$$

Thus, since

$$
m-\sum_{j=2}^{r} n_{j} \in\left(b_{2}, \cdots, b_{r}\right) M: b_{1}^{n}=\left(b_{2}, \cdots, b_{r}\right) M: \mathfrak{b},
$$

$m \in \sum_{\jmath=1}^{r}\left(b_{1}, \cdots, b_{j}, \cdots, b_{r}\right) M: \mathfrak{b}$, as required.

Theorem 3.4 has many interesting consequences.

CoRollary 3.6 (cf. [23, Theorem 1]). a is M-standard if the natural homomorphism

$$
\phi_{i}: \operatorname{Ext}_{A}^{i}(A / \mathfrak{a}, M) \longrightarrow H_{\mathrm{m}}^{i}(M)
$$

is surjective for $i=0, \cdots, d-1$. If $a$ is generated by a regular $A$-sequence, the converse also holds.

Proof. The first statement is easily seen from the following commutative diagram

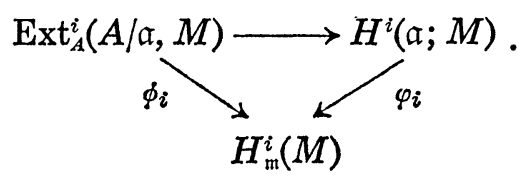


For the second statement we only need to note that if $a$ is generated by a regular $A$-sequence, then $\operatorname{Ext}_{A}^{i}(A / \mathfrak{a}, M) \widetilde{\rightarrow} H^{i}(\mathfrak{a} ; M)$.

Corollary 3.7 (cf. [23], Corollary 1.1]). Suppose that there exists a non-negative integer $r<d$ such that $H_{\mathrm{tm}}^{i}(M)=0$ for $i \neq r, d$. Then $a$ is $M$-standard iff $\mathfrak{a} H_{\mathrm{m}}^{r}(M)=0$.

Proof. It is sufficient to prove the sufficient part. First, we have

$$
\begin{aligned}
& H^{r}(\mathfrak{a} ; M) \cong H^{0}\left(\mathfrak{a} ; M /\left(a_{1}, \cdots, a_{r}\right) M\right)=\left(a_{1}, \cdots, a_{r}\right) M: a /\left(a_{1}, \cdots, a_{r}\right) M \\
& H_{\mathrm{m}}^{r}(M) \cong H_{\mathfrak{m}}^{0}\left(M /\left(a_{1}, \cdots, a_{r}\right) M\right)=\bigcup_{n=1}^{\infty}\left(a_{1}, \cdots, a_{r}\right) M: \mathfrak{a}^{n} /\left(a_{1}, \cdots, a_{r}\right) M
\end{aligned}
$$

for some regular $M$-sequence $a_{1}, \cdots, a_{r}$ in $a$. Since $a H_{\mathrm{m}}^{r}(M)=0$, we must have

$$
\left(a_{1}, \cdots, a_{r}\right) M: \mathfrak{a}=\bigcup_{n=1}^{\infty}\left(a_{1}, \cdots, a_{r}\right) M: \mathfrak{a}^{n},
$$

hence $H^{r}(\mathfrak{a} ; M) \cong H_{\mathrm{m}}^{r}(M)$.

Further, we can show that there exists a practical reduction process to check where a given ideal is $M$-standard.

First, we note that by Corollary 2.4, $\mathfrak{a}$ is $M / a M$-standard for every element $a \in \mathfrak{a}$ which forms part of a s.o.p. of $M$, porvided that $a$ is $M$ standard. However, the existence of such an element $a$ does not imply (even in the case depth $M>0$ ) that $a$ is $M$-standard, see [31]. But we still have the following non-zerodivisor characterization of standard ideals in case depth $M>0$ :

Corollary 3.8 (cf. [24, Satz 6.5] or [31, Theorem]). Suppose that depth $M>0$. Then $\mathfrak{a}$ is $M$-standard iff $\mathfrak{a}$ is $M / a M$-standard for some non-zerodivisor $a \in \mathfrak{a}$ of $M$ with one of the following two properties:

(i) $a \in \mathfrak{a}^{2}$.

(ii) $a H_{\mathrm{m}}^{i}(M)=0$ for all $i=1, \cdots, d-1$.

The case depth $M=0$ can be transferred to the case depth $M>0$ by the following result:

Corollary 3.9 (cf. [25, Theorem 4]). a is M-standard iff the following conditions are satisfied:

(i) a is $\bar{M}$-standard.

(ii) $\left(a_{1}, \cdots, a_{d}\right) M \subset H_{\mathrm{m}}^{0}(M)=0$ for every $d$ element subset. $\left\{a_{1}, \cdots, a_{d}\right\}$ of some $M$-base of a. 
The proofs for Corollaries 3.8 and 3.9 (based on Theorem 3.4) are similar to the ones for [24, Satz 6.5], [25, Theorem 4]. Hence we omit them.

For the remainder of this section, let $R=\bigoplus_{n=0}^{\infty} R_{n}$ be a noetherian graded ring such that $R_{0}$ is a local ring. Note that with respect to the theory of graded modules, $R$ behaves as though it were local. Then we say that a graded structure over $R$ will have some property (C) which can be only formulated over local rings if (C) holds for the corresponded structure over $R_{P}$, where $P$ denotes the maximal graded ideal of $R$. So we can use the notions of (homogeneous) generalized C-M modules, standard s.o.p.'s, standard ideals, etc. over $R$.

Let $E$ be a finitely generated graded $R$-module with $d:=\operatorname{dim} E \geqq 1$ and $I$ a graded ideal contained in the ideal $R^{+}$of elements of positive degree of $R$ with $l(E / I E)<\infty$. Put

$$
N_{i}:=\left\{n ;\left[H_{P}^{i}(E)\right]_{n} \neq 0\right\}
$$

for $i=0, \cdots, d-1$. Then under certain assumption on $N_{i}$, we can give a criterion for $I$ to be a $E$-standard ideal.

Theorem 3.10. Suppose that $E$ is a generalized $C$ - $M$ module with

$$
\max N_{i} \leqq \min N_{i+1}+1 \text {, }
$$

$i=0, \cdots, d-2$. Then $I$ is a E-standard ideal iff

$$
I H_{P}^{i}(E)=0
$$

for $i=0, \cdots, d-1$.

For the proof of Theorem 3.10 we shall need the following

LEMMA 3.11. Let $a_{1}, \cdots, a_{d}$ be a system of homogeneous parameters of $E$ of positive degree. Then, by the assumption of Theorem 3.10, there exists a graded homomorphism

$$
\varphi_{j}^{i}: H_{P}^{i}\left(E / \mathfrak{q}_{j} E\right) \longrightarrow H_{P}^{i+j}(E)
$$

of degree $t_{j}:=-\sum_{t=1}^{j} \operatorname{deg}\left(a_{t}\right)$ which is injective in degree $>\min N_{i+j}-t_{j}$ for all non-negative integers $i, j$ with $i+j<d$.

Proof. We go by induction on $j$. For $j=0$ there is nothing to prove. For $j>0$ we consider the exact sequence 


$$
0 \longrightarrow E / \mathfrak{q}_{j-1} E: a_{j} \longrightarrow E / \mathfrak{q}_{j-1} E \longrightarrow E / \mathfrak{q}_{j} E \longrightarrow 0 \text {. }
$$

Note that by Lemma 1.1 (iv), $\mathfrak{q}_{j-1} E: a_{j} / \mathfrak{q}_{j-1} E \subseteq H_{P}^{0}\left(E / \mathfrak{q}_{j-1} E\right)$ is of finite length. Then the natural homomorphism

$$
H_{P}^{i+1}\left(E / \mathfrak{q}_{j-1} E\right) \longrightarrow H_{P}^{i+1}\left(E / \mathfrak{q}_{j-1} E: a_{j}\right)
$$

is an isomorphism. Hence we have an exact sequence

$$
H_{P}^{i}\left(E / \mathfrak{q}_{j-1} E\right) \stackrel{\alpha}{\longrightarrow} H_{P}^{i}\left(E / \mathfrak{q}_{j} E\right) \stackrel{\beta}{\longrightarrow} H_{P}^{i+1}\left(E / \mathfrak{q}_{j-1} E\right)
$$

with $\operatorname{deg} \alpha=0$ and $\operatorname{deg} \beta=-\operatorname{deg}\left(a_{j}\right)$. Put

$$
\varphi_{j}^{i}=\varphi_{j-1}^{i+1} \beta
$$

where the existence of $\varphi_{j-1}^{i+1}$ follows from the induction hypothesis. Then $\varphi_{j}^{i}$ is a graded homomorphism from $H_{P}^{i}\left(E / \mathfrak{q}_{j} E\right)$ to $H_{P}^{i+j}(E)$ of degree $t_{j}=$ $t_{j-1}-\operatorname{deg}\left(a_{j}\right)$. To show that $\varphi_{j}^{i}$ is injective in degree $>\min N_{i+j}-t_{j}$, we only need to show that $\left[H_{P}^{i}\left(E / \mathfrak{q}_{j-1} E\right)\right]_{n}=0$ for $n>\min N_{i+j}-t_{j}$. Note that

$$
\begin{aligned}
\min N_{i+j}-t_{j} & \geqq \max N_{i+j-1}-1-t_{j-1}+\operatorname{deg}\left(a_{j}\right) \\
& \geqq \max N_{i+j-1}-t_{j-1} .
\end{aligned}
$$

Then $\left[H_{P}^{i+j-1}(E)\right]_{n+t_{j-1}}=0$ by the definition of $N_{i+j-1}$, hence so is $\left[H_{P}^{i}\left(E / \mathfrak{q}_{j} E\right)\right]_{n}$ as a submodule of $\left[H_{P}^{i+j-1}(E)\right]_{n+t_{j-1}}$ by the induction hypothesis on $\varphi_{j-1}^{i}$.

Proof of Theorem 3.10. It suffices to show the sufficient part. Suppose that $I H_{P}^{i}(E)=0$ for $i=0, \cdots, d-1$. Then we want to show that every homogeneous s.o.p. $a_{1}, \cdots, a_{d}$ of $E$ contained in $I$ is a $d$-sequence of $E$, which then implies that $I$ is a $E$-standard ideal by Proposition 3.1. By Remark 2.8 (2), we only need to show that

$$
\mathfrak{q}_{i-1} E: a_{i}^{2} \subseteq \mathfrak{q}_{i-1} E: \mathfrak{q}
$$

for $i=1, \cdots, d$. First, by Lemma 1.1 (iv), we have

$$
\mathfrak{q}_{i-1} E: a_{i}^{2} / \mathfrak{q}_{i-1} E \subseteq H_{P}^{0}\left(E / \mathfrak{q}_{i-1} E\right)
$$

Hence the case $i=1$ immediately follows from the fact $\mathfrak{q} H_{P}^{0}(E) \subseteq I H_{P}^{0}(E)$ $=0$. Now let $i>1$. Since $I$ contains only elements of positive degree, from Lemma 3.11 we can easily see that via the homomorphism $\varphi_{i-1}^{0}$,

$$
\mathfrak{q}\left[\mathfrak{q}_{i-1} E: a_{i}^{2} / \mathfrak{q}_{i-1} E\right]_{t} \subseteq I\left[H_{P}^{0}\left(E / \mathfrak{q}_{i-1} E\right)\right]_{t} \subseteq I H_{P}^{i-1}(E)=0
$$


for $t \geqq \min N_{i-1}-t_{i-1}$. It remains to show that

$$
\left[\mathfrak{q}_{i-1} E: a_{i}^{2}\right]_{t} \subseteq \mathfrak{q}_{i-1} E: \mathfrak{q}
$$

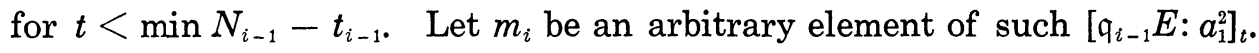
Then

$$
a_{i}^{2} m_{i}=-\sum_{j=1}^{i-1} a_{j} m_{j}
$$

for some $m_{j} \in E$. Thus, $m_{1}, \cdots, m_{i}$ define a cycle of $K\left(a_{1}, \cdots, a_{i-1}, a_{i}^{2} ; E\right)$

$$
e=\sum_{j=1}^{i} m_{j} X_{j}
$$

with

$$
\operatorname{deg}(e)=t-\sum_{j=1}^{i-1} \operatorname{deg}\left(a_{j}\right)=t+t_{i-1}<\min N_{i-1} .
$$

Note that by the proof for the sufficient part of Theorem 3.4, we have a surjective homomorphism of degree 0 from $H_{P}^{i-1}(E)$ onto $H_{9 i}^{i-1}(E)$. Then $H_{q i}^{i-1}(E)=0$ in degree $<\min N_{i-1}$. Hence there exist integers $n$ such that the image of $e$ in $K\left(a_{1}^{n}, \cdots, a_{i}^{n} ; E\right)$ is a boundary. From this it follows that

$$
\left(a_{1} \cdots a_{i-1}\right)^{n-1} m_{i} \in\left(a_{1}^{n}, \cdots, a_{i-1}^{n}\right) E .
$$

Now, proceeding as at the end of the proof for the sufficient part of Theorem 3.4, we get

$$
m_{i} \in\left(a_{1}^{n}, \cdots, a_{i-1}^{n}\right) E:\left(a_{1} \cdots a_{i-1}\right)^{n-1} \subseteq \mathfrak{q}_{i-1} E: \mathfrak{q},
$$

as required. The proof of Theorem 3.10 is now complete.

From Theorem 3.10 we immediately get the following consequence which generalizes a well-known criterion for graded Buchsbaum rings.

Corollary 3.12 (cf. [8, Proposition 3.1]). Suppose that there exist integers $t_{0}, \cdots, t_{d-1}$ with $t_{i} \leqq t_{i+1}+1, i=0, \cdots, d-2$, such that

$$
\left[H_{P}^{i}(E)\right]_{n}=0
$$

for $n \neq t_{i}, i=0, \cdots, d-1$. Then $R^{+}$is an E-standard ideal.

\section{§4. Hilbert-Samuel functions}

Throughout this section, $M$ will be a generalized C-M module.

In [27, Theorem 4.1] we showed that the Hilbert-Samuel (abbr. H-S) 
function $l\left(M / \mathfrak{q}^{n+1} M\right)$ of $M$ relative to a parameter ideal $\mathfrak{q}=\left(a_{1}, \cdots, a_{d}\right)$ is bounded above by a polynomial of the form

$$
\sum_{i=0}^{d}\left(\begin{array}{c}
n+d-i \\
d-i
\end{array}\right) e_{i}(\mathfrak{q} ; M)
$$

where $e_{i}(\mathfrak{q} ; M)$ may be expressed explicitly in terms of $a_{1}, \cdots, a_{d}$, and that they coincide iff $a_{1}, \cdots, a_{d}$ is a $d$-sequence of $M$. Now we shall show a similar but stronger result for generalized C-M modules.

TheOREM 4.1. Let $a_{1}, \cdots, a_{d}$ be an arbitrary s.o.p. of $M$. Then

$$
l\left(M / \mathfrak{q}^{n+1} M\right) \leqq\left(\begin{array}{c}
n+d \\
d
\end{array}\right) e(\mathfrak{q} ; M)+\sum_{i=1}^{d} \sum_{j=0}^{d-i}\left(\begin{array}{c}
n+d-i \\
d-i
\end{array}\right)\left(\begin{array}{c}
d-i-1 \\
j-1
\end{array}\right) l\left(H_{\mathrm{m}}^{j}(M)\right)
$$

for all $n \geqq 0$, where $\left(\begin{array}{c}d-i-1 \\ -1\end{array}\right):=0$ if $i \neq d$ and $\left(\begin{array}{l}-1 \\ -1\end{array}\right):=1$. Equality holds for some fixed $n$ iff the following conditions are satisfied:

(i) $\mathfrak{q}^{n+1} M \cap H_{\mathrm{m}}^{0}(M)=0$.

(ii) $a_{1}, \cdots, a_{d}$ is a standard s.o.p. of $\bar{M}$.

Proof. We first consider the case depth $M>0$. Let $M_{1}$ denote the factor module $M / a_{1} M$. Then we have the following exact sequence:

$$
0 \longrightarrow \mathfrak{q}^{t+1} M ; a_{1} / \mathfrak{q}^{t} M \longrightarrow M / \mathfrak{q}^{t} M \stackrel{a_{1}}{\longrightarrow} M / \mathfrak{q}^{t+1} M \longrightarrow M_{1} / \mathfrak{q}^{t+1} M_{1} \longrightarrow 0
$$

for all $t \geqq 0$. From this sequence we get

$$
l\left(\mathfrak{q}^{t} M / \mathfrak{q}^{t+1} M\right) \leqq l\left(M_{1} / \mathfrak{q}^{t+1} M_{1}\right)
$$

By induction on $d$ (we include the case $d=0$ which is trivial), we may assume that

$$
\begin{aligned}
l\left(M_{1} / \mathfrak{q}^{t+1} M_{1}\right) & \leqq\left(\begin{array}{c}
t+d-1 \\
d-1
\end{array}\right) e\left(a_{2}, \cdots, a_{d} ; M_{1}\right) \\
& +\sum_{i=1}^{d-1} \sum_{j=0}^{d-i-1}\left(\begin{array}{c}
t+d-i-1 \\
d-i-1
\end{array}\right)\left(\begin{array}{c}
d-i-2 \\
j-1
\end{array}\right) l\left(H_{\mathrm{m}}^{j}(M)\right) .
\end{aligned}
$$

Since $e\left(a_{2}, \cdots, a_{d} ; M_{1}\right)=e(\mathfrak{q} ; M)$ and, by Lemma 1.7 (i),

$$
l\left(H_{\mathrm{m}}^{j}\left(M_{1}\right)\right) \leqq l\left(H_{\mathrm{m}}^{j}(M)\right)+l\left(H_{\mathrm{m}}^{j+1}(M)\right),
$$

we get 


$$
\begin{aligned}
& l\left(\mathfrak{q}^{t} M / \mathfrak{q}^{t+1} M\right) \leqq\left(\begin{array}{c}
t+d-1 \\
d-1
\end{array}\right) e(\mathfrak{q} ; M) \\
& \quad+\sum_{i=1}^{d-1} \sum_{j=0}^{d-i-1}\left(\begin{array}{c}
t+d-i-1 \\
d-i-1
\end{array}\right)\left(\begin{array}{c}
d-i-2 \\
j-1
\end{array}\right)\left[l\left(H_{\mathrm{m}}^{j}(M)\right)+l\left(H_{\mathfrak{m}}^{j+1}(M)\right)\right] \\
& \quad=\left(\begin{array}{c}
t+d-1 \\
d-1
\end{array}\right) e(\mathfrak{q} ; M)+\sum_{i=1}^{d} \sum_{j=0}^{d-i}\left(\begin{array}{c}
t+d-i-1 \\
d-i-1
\end{array}\right)\left(\begin{array}{c}
d-i-1 \\
j-1
\end{array}\right) l\left(H_{\mathfrak{m}}^{j}(M)\right)
\end{aligned}
$$

(we omit a detailed calculation here and below). Thus,

$$
\begin{aligned}
& l\left(M / \mathfrak{q}^{n+1} M\right)= \sum_{t=0}^{n} l\left(\mathfrak{q}^{t} M / \mathfrak{q}^{t+1} M\right) \leqq \sum_{i=0}^{n}\left(\begin{array}{c}
t+d-1 \\
d-1
\end{array}\right) e(\mathfrak{q} ; M) \\
&+\sum_{t=0}^{n} \sum_{i=1}^{d} \sum_{j=0}^{d-1}\left(\begin{array}{c}
t+d-i-1 \\
d-i-1
\end{array}\right)\left(\begin{array}{c}
d-i-1 \\
j-1
\end{array}\right) l\left(H_{\mathfrak{m}}^{j}(M)\right) \\
&=\left(\begin{array}{c}
n+d \\
d
\end{array}\right) e(\mathfrak{q} ; M)+\sum_{i=1}^{d} \sum_{j=0}^{d-i}\left(\begin{array}{c}
n+d-i \\
j-1
\end{array}\right)\left(\begin{array}{c}
d-i-1 \\
j-1
\end{array}\right) l\left(H_{\mathrm{m}}^{j}(M)\right) .
\end{aligned}
$$

In particular, according to (3), equality for some $n \geqq 0$ will imply

$$
\begin{aligned}
l(M / \mathfrak{q} M) & =e(\mathfrak{q} ; M)+\sum_{i=1}^{d} \sum_{j=0}^{d-i}\left(\begin{array}{l}
d-i-1 \\
d-i-1
\end{array}\right)\left(\begin{array}{c}
d-i-1 \\
j-1
\end{array}\right) l\left(H_{\mathrm{m}}^{j}(M)\right) \\
& =e(\mathfrak{q} ; M)+I(M)
\end{aligned}
$$

by Lemma 1.5. Hence $a_{1}, \cdots, a_{d}$ is a standard s.o.p. of $M$.

For the case depth $M=0$, we pass to $\bar{M}$ (depth $\bar{M}>0$ ) as follows:

$$
\begin{aligned}
l\left(M / \mathfrak{q}^{n+1} M\right)= & l\left(M / \mathfrak{q}^{n+1} M+H_{\mathrm{m}}^{0}(M)\right)+l\left(\mathfrak{q}^{n+1} M+H_{\mathrm{m}}^{0}(M) / \mathfrak{q}^{n+1} M\right) \\
= & l\left(\bar{M} / \mathfrak{q}^{n+1} \bar{M}\right)+l\left(H_{\mathrm{m}}^{0}(M) / \mathfrak{q}^{n+1} M \cap H_{\mathrm{m}}^{0}(M)\right) \\
= & \left(\begin{array}{c}
n+d \\
d
\end{array}\right) e(\mathfrak{q} ; \bar{M})+\sum_{i=1}^{d} \sum_{j=0}^{d-i}\left(\begin{array}{c}
n+d-i \\
d-i
\end{array}\right)\left(\begin{array}{c}
d-i-1 \\
j-1
\end{array}\right) l\left(H_{\mathrm{m}}^{j}(\bar{M})\right) \\
& +l\left(H_{\mathrm{m}}^{0}(M)\right) \\
= & \left(\begin{array}{c}
n+d \\
d
\end{array}\right) e(\mathfrak{q} ; M)+\sum_{i=1}^{d} \sum_{j=0}^{d-i}\left(\begin{array}{c}
n+d-i \\
d-i
\end{array}\right)\left(\begin{array}{c}
d-i-1 \\
j-1
\end{array}\right) l\left(H_{\mathrm{m}}^{j}(M)\right)
\end{aligned}
$$

because $e(\mathfrak{q} ; \bar{M})=e(\mathfrak{q} ; M), H_{\mathrm{m}}^{0}(\bar{M})=0, H_{\mathrm{m}}^{j}(\bar{M})=H_{\mathrm{m}}^{j}(M)$ for $j=1, \cdots$, $d-1$. From this proof we can easily see that equality in (5) implies the conditions (i) and (ii).

Conversely, assume that (i) and (ii) are satisfied. Then using (5), we may assume that depth $M>0$. By Corollary 2.6 (v), we have

$$
\mathfrak{q}^{t+1} M: a_{1}=\mathfrak{q}^{t} M
$$

for all $t \geqq 0$. Hence (1) is an equality. By induction, we may also assume 
that (2) is an equality. Further, since by Theorem $2.5 a_{1} H_{\mathrm{m}}^{j}(M)=0$ for all $j=0, \cdots, d-1$, we have

$$
l\left(H_{\mathrm{m}}^{j}\left(M_{1}\right)\right)=l\left(H_{\mathrm{m}}^{j}(M)\right)+l\left(H_{\mathrm{m}}^{j+1}(M)\right)
$$

for $j=0, \cdots, d-2$ by Lemma 1.7 . Hence (3) is an equality too. Thus, (4) is an equality, as required.

From Corollary 2.3 and Theorem 4.1 we immediately get the following consequence which generalizes the main result of [18].

Corollary 4.2. $a_{1}, \cdots, a_{d}$ is a standard s.o.p. of $M$ iff

$$
l\left(M / \mathfrak{q}^{n+1} M\right)=\left(\begin{array}{c}
n+d \\
d
\end{array}\right) e(\mathfrak{q} ; M)+\sum_{i=1}^{d} \sum_{j=0}^{d-i}\left(\begin{array}{c}
n+d-i \\
d-i
\end{array}\right)\left(\begin{array}{c}
d-i-1 \\
j-1
\end{array}\right) l\left(H_{\mathfrak{m}}^{j}(M)\right)
$$

for all $n \geqq 0$.

Now we will use Theorem 4.1 to study the H-S function of an arbitrary submodule $N$ of $M$ with $l(M / N)<\infty$ relative to an ideal a of $A$ with $l(M / \mathfrak{a} M)<\infty$.

We shall need the following notion of [27]:

Definition. $\quad a_{1}, \cdots, a_{d}$ is called $N$-independent if every homogeneous form in $d$ indeterminates over $M$ vanishing at $a_{1}, \cdots, a_{d}$ has all its coefficients in $N$.

If $a_{1}, \cdots, a_{d}$ is a standard s.o.p. of $M$, one can use the following result to check whether $a_{1}, \cdots, a_{d}$ is $N$-independent:

Lemma 4.3 [27, Corollary 3.4]. Let $a_{1}, \cdots, a_{d}$ be a $d$-sequence of $M$. Then the following conditions are equivalent:

(i ) $a_{1}, \cdots, a_{d}$ are $N$-independent.

(ii) $\mathfrak{q}_{d-1} M: a_{d} \subseteq N$ by every permutation of $a_{1}, \cdots, a_{d}$.

(iii) $l\left(\mathfrak{q}^{n} M / \mathfrak{q}^{u} N\right)=\left(\begin{array}{c}n+d-1 \\ d-1\end{array}\right) l(M / N)$ for some (or all) $n \geqq 1$.

Moreover, we call $a_{1}, \cdots, a_{d}$ a minimal reduction of a relative to $N$ if $a_{1}, \cdots, a_{d} \in \mathfrak{a} \mid \mathfrak{a}^{2}$ and their initial forms in $G_{a}(A)$ forms a homogeneous s.o.p. of $G_{a}(N)$. In this case, we have

$$
e(\mathfrak{q} ; N)=e(\mathfrak{a} ; N) \text {. }
$$

It is well-known that minimal reductions always exist if the residue field $k:=A / \mathfrak{m}$ is infinite, a hypothesis which never cause us any problem because we can replace $A$ by the local ring $A[u]_{\mathrm{m}[u]}$, where $u$ is some indeterminate. See [16]. 
Our result concerning the $\mathrm{H}-\mathrm{S}$ function of $N$ relative to $\mathfrak{a}$ may be formulated as follows.

Proposition 4.4. Let $N$ and $\mathfrak{a}$ be as above. Then $l\left(M / \mathfrak{a}^{n} N\right)$

$$
\begin{aligned}
\leqq & \left(\begin{array}{c}
n+d-1 \\
d
\end{array}\right) e(\mathfrak{a} ; M)+\sum_{i=1}^{d} \sum_{j=0}^{d-i}\left(\begin{array}{c}
n+d-i-1 \\
d-i
\end{array}\right)\left(\begin{array}{c}
d-i-1 \\
j-1
\end{array}\right) l\left(H_{\mathrm{m}}^{j}(M)\right) \\
& +\left(\begin{array}{c}
n+d-1 \\
d-1
\end{array}\right) l\left(M / N+H_{\mathrm{m}}^{0}(M)\right)
\end{aligned}
$$

for all $n \geqq 1$. If $k$ is infinite, equality holds for some fixed $n$ iff for every or some minimal reduction $a_{1}, \cdots, a_{d}$ of a relative to $N$, the following conditions are satisfied:

(i) $\mathfrak{q}^{n} N=\mathfrak{a}^{n} N$.

(ii) $a_{1}, \cdots, a_{d}$ is a standard s.o.p. of $\bar{M}$.

(iii) $\left[\mathfrak{q}_{d-1} M+H_{\mathrm{m}}^{0}(M)\right]: a_{d} \subseteq N+H_{\mathrm{m}}^{0}(M)$ by every permutation of $a_{1}$, $\cdots, a_{d}$.

(iv) $\mathfrak{q}^{n} N \cap H_{\mathrm{m}}^{0}(M)=0$.

Proof. Without restriction we may assume that $k$ is infinite. Then there exists a minimal reduction $a_{1}, \cdots, a_{d}$ of $a$ relative to $N$. We have

$$
\begin{aligned}
l\left(M / \mathfrak{a}^{n} N\right) \leqq & l\left(M / \mathfrak{q}^{n} N\right)=l\left(M / \mathfrak{q}^{n} M+H_{\mathrm{m}}^{0}(M)\right)+l\left(\mathfrak{q}^{n} M+H_{\mathrm{m}}^{0}(M) / \mathfrak{q}^{n} M\right) \\
& \left.+l\left(\mathfrak{q}^{n} M / \mathfrak{q}^{n} N+\mathfrak{q}^{n} H_{\mathrm{m}}^{0}(M)\right)+l\left(\mathfrak{q}^{n} N+\mathfrak{q}^{n} H_{\mathrm{m}}^{0} / M\right) / \mathfrak{q}^{n} .\right) .
\end{aligned}
$$

By Theorem 4.1, it is easy to verify that

$$
\begin{aligned}
l\left(M / \mathfrak{q}^{n} M+\right. & \left.H_{\mathrm{m}}^{0}(M)\right)=l\left(\bar{M} / \mathfrak{q}^{n} \bar{M}\right) \leqq\left(\begin{array}{c}
n+d-1 \\
d
\end{array}\right) e(\mathfrak{a} ; M) \\
& +\sum_{i=1}^{d} \sum_{j=1}^{d-i}\left(\begin{array}{c}
n+d-i-1 \\
d-i
\end{array}\right)\left(\begin{array}{c}
d-i-1 \\
j-1
\end{array}\right) l\left(H_{\mathrm{m}}^{j}(M)\right) .
\end{aligned}
$$

Further, we have

$$
\begin{aligned}
& l\left(\mathfrak{q}^{n} M+H_{\mathrm{m}}^{0}(M) / \mathfrak{q}^{n} M\right)+l\left(\mathfrak{q}^{n} N+\mathfrak{q}^{n} H_{\mathrm{m}}^{0}(M) / \mathfrak{q}^{n} N\right) \\
& \quad=l\left(H_{\mathrm{m}}^{0}(M) / \mathfrak{q}^{n} M \cap H_{\mathrm{m}}^{0}(M)\right)+l\left(\mathfrak{q}^{n} H_{\mathrm{m}}^{0}(M) / \mathfrak{q}^{n} N \cap \mathfrak{q}^{n} H_{\mathrm{m}}^{0}(M)\right) \\
& \quad \leqq l\left(H_{\mathrm{m}}^{0}(M) / \mathfrak{q}^{n} N \cap \mathfrak{q}^{n} H_{\mathrm{m}}^{0}(M)\right) \leqq l\left(H_{\mathrm{m}}^{0}(M)\right) .
\end{aligned}
$$

Moreover, since $\mathfrak{q}^{n}$ is generated by $\left(\begin{array}{c}n+d-1 \\ d-1\end{array}\right)$ monomials of degree $n$ in $a_{1}, \cdots, a_{d}$,

$$
l\left(\mathfrak{q}^{n} M / \mathfrak{q}^{n} N+\mathfrak{q}^{n} H_{\mathrm{m}}^{0}(M)\right) \leqq\left(\begin{array}{c}
n+d-1 \\
d-1
\end{array}\right) l\left(M / N+H_{\mathrm{m}}^{0}(M)\right) .
$$

Hence, summing up, we get 
$l\left(M / a^{n} M\right)$

$$
\begin{gathered}
\leqq\left(\begin{array}{c}
n+d-1 \\
d
\end{array}\right) e(a ; M)+\sum_{i=1}^{d} \sum_{j=0}^{d-i}\left(\begin{array}{c}
n+d-i-1 \\
d-1
\end{array}\right)\left(\begin{array}{c}
d-i-1 \\
j-1
\end{array}\right) l\left(M / \mathfrak{a}^{n} M\right) \\
+\left(\begin{array}{c}
n+d-1 \\
d
\end{array}\right) l\left(M / N+H_{\mathrm{m}}^{0}(M)\right) .
\end{gathered}
$$

Equality holds above iff we have equalities in (1) to (4). Clearly, (1) is an equality iff (i) is satisfied. By Theorem 4.1, (2) is an equality iff (ii) is satisfied. (3) is an equality iff

$$
\mathfrak{q}^{n} M \cap H_{\mathrm{m}}^{0}(M)=\mathfrak{q}^{n} H_{\mathrm{m}}^{0}(M) \quad \text { and } \quad \mathfrak{q}^{n} N \cap \mathfrak{q}^{n} H_{\mathrm{m}}^{0}(M)=0 .
$$

By Lemma 4.3, (4) is an equality iff (iii) is satisfied, provided that $a_{1}$, $\cdots, a_{d}$ is a standard s.o.p. of $\bar{M}$. Thus, to prove the last statement of Proposition 4.4, we only need to show that (5) is equivalent to (iv) under the assumption that (ii) and (iii) are already satisfied. It suffices to show that (iv) implies

$$
\mathfrak{q}^{n} M \cap H_{\mathrm{m}}^{0}(M)=q^{n} H_{\mathrm{m}}^{0}(M) .
$$

Since $a_{1}, \cdots, a_{d}$ are $\left(N+H_{\mathrm{m}}^{0}(M) / H_{\mathrm{m}}^{0}(M)\right)$-independent by Lemma 4.3 , we first have

$$
\mathfrak{q}^{n} M \cap H_{\mathrm{m}}^{0}(M)=\mathfrak{q}^{n}\left(N+H_{\mathrm{m}}^{0}(M)\right) \cap H_{\mathrm{m}}^{0}(M) .
$$

Hence, applying (iv), we get $\mathfrak{q}^{n} M \cap H_{\mathrm{m}}^{0}(M)=\mathfrak{q}^{n} H_{\mathrm{m}}^{0}(M)$, as required. The proof of Proposition 4.4 is now complete.

Corollary 4.5. Suppose that the inequalily of Proposition 4.4 is an equality for some $n \geqq 1$. Then $\mathfrak{a}$ is a standard ideal of $\bar{M}$.

Proof. By Theorem 3.4, we may assume that $k$ is an infinite field. Then there exists a generating set $S$ for a such that every $d$ element subset of $S$ forms a minimal reduction of a relative to $N$. Hence, applying Proposition 3.2 and Proposition 4.4, we get the statement.

From Proposition 4.4 we get the following estimation of the $\mathrm{H}-\mathrm{S}$ function of $M$ relative to a:

COROLLARY 4.6 (cf. [26, Lemma 1.1]). Let $r \geqq 0$ and $s \geqq 1$ be arbitrary integers. Then

$l\left(M / \mathfrak{a}^{n s+r} M\right)$

$$
\begin{aligned}
\leqq & \left(\begin{array}{c}
n+d-1 \\
d
\end{array}\right) s^{d} e(\mathfrak{a} ; M)+\sum_{i=1}^{d} \sum_{j=0}^{d-i}\left(\begin{array}{c}
n+d-i-1 \\
d-i
\end{array}\right)\left(\begin{array}{c}
d-i-1 \\
j-1
\end{array}\right) l\left(H_{\mathrm{m}}^{j}(M)\right) \\
& +\left(\begin{array}{c}
n+d-1 \\
d-1
\end{array}\right) l\left(M / \mathfrak{a}^{r} M+H_{\mathrm{m}}^{0}(M)\right)
\end{aligned}
$$


for all $n \geqq 1$. Moreover, if $r>s$, the inequality is proper.

Proof. For the first statement, we only need to replace $a$ and $N$ of Proposition 4.4 by $\mathfrak{a}^{s}$ and $\mathfrak{a}^{r} M$. If $r>s$, equality can not happen. Otherwise, we have

$$
\mathfrak{q}_{d-1} M \subseteq\left(\mathfrak{q}_{d-1} M+H_{\mathrm{m}}^{0}(M)\right): a_{d} \subseteq \mathfrak{a}^{r} M+H_{\mathrm{m}}^{0}(M)
$$

for every minimal reduction $a_{1}, \cdots, a_{d}$ of $\mathfrak{a}^{s}$ relative to $\mathfrak{a}^{r} M$ by Proposition 4.4 (iii). Since we may assume that $k$ is infinite, there exists a generating set $S$ for $\mathfrak{a}^{s}$ such that every $d$ element subset of $S$ forms a minimal reduction of $\mathfrak{a}^{s}$ relative to $\mathfrak{a}^{r} M$. Hence we must have

$$
\mathfrak{a}^{s} M \subseteq \mathfrak{a}^{r} M+H_{\mathfrak{m}}^{0}(M) \subseteq \mathfrak{m a}^{s} M+H_{\mathfrak{m}}^{0}(M),
$$

which then implies that $\mathfrak{a}^{s} M=H_{\mathrm{m}}^{0}(M)$, a contradiction to the assumption $d \geqq 1$.

Corollary 4.6 has the following interesting consequence which may be used to study the relationship between degree, genus, and local cohomology of a projective curve.

Corollary 4.7. Let $M$ be a two-dimensional generalized $C$ - $M$ module. Let $H(n)=l\left(M / \mathfrak{a} M^{n+1}\right)$ denote the Hilbert-Samuel polynomial of $M$ with respect to $\mathfrak{a}$. Then

$$
H(n)+l\left(H_{\mathrm{m}}^{1}(M)\right)-l\left(H_{\mathrm{m}}^{0}(M)\right) \geqq 0
$$

for all integers $n$. Moreover, if $n<0$, the inequality is proper.

Proof. Put

$$
H(n):=\frac{n(n+1)}{2} e_{0}+n e_{1}+e_{2} .
$$

Note that

$$
\begin{aligned}
l\left(M / \mathfrak{a}^{r} M+H_{\mathrm{m}}^{0}(M)\right) & =l\left(M / \mathfrak{a}^{r} M\right)-l\left(\mathfrak{a}^{r} M+H_{\mathrm{m}}^{0}(M) / \mathfrak{a}^{r} M\right) \\
& =l\left(M / \mathfrak{a}^{r} M\right)-l\left(H_{\mathrm{m}}^{0}(M) / \mathfrak{a}^{r} M \cap H_{\mathrm{m}}^{0}(M)\right) \\
& =H(r)-l\left(H_{\mathrm{m}}^{0}(M)\right)
\end{aligned}
$$

for $r$ sufficiently large. Then, using Corollary 4.6, we have

$$
\begin{aligned}
& \frac{(n s+r)(n s+r+1)}{2} e_{0}+(n s+r) e_{1}+e_{2} \\
& \leqq \frac{n(n+1)}{2} s^{2} e_{0}+n l\left(H_{\mathrm{m}}^{1}(M)\right)+l\left(H_{\mathrm{m}}^{0}(M)\right) \\
& \quad+(n+1)\left[\frac{r(r+1)}{2} e_{0}+r e_{1}+e_{2}-l\left(H_{\mathrm{m}}^{0}(M)\right)\right]
\end{aligned}
$$


for arbitrary $s, n \geqq 1$. From this it follows, by elementary computation, that

$$
\frac{(r-s)(r-s+1)}{2} e_{0}+(r-s) e_{1}+e_{2}+l\left(H_{\mathrm{m}}^{1}(M)\right)-l\left(H_{\mathrm{m}}^{0}(M)\right) \geqq 0 .
$$

Since $r-s$ can take any value, we get the statement.

For $n=1$, the inequality of Proposition 4.4 yields

$$
l(M / \mathfrak{a} N) \leqq e(\mathfrak{a} ; M)+I(M)+d l\left(M / N+H_{\mathrm{m}}^{0}(M)\right) .
$$

From this it follows, for $M=A$ and $N=\mathfrak{a}=\mathfrak{m}$, that

$$
l\left(\mathfrak{m} / \mathfrak{m}^{2}\right) \leqq e(\mathfrak{m} ; A)+I(A)+d-1
$$

which gives a bound for the embedding dimension of generalized C-M rings, cf. [1], [17], [26]. If this bound is attained, we call $A$ a local ring of maximal embedding dimension. J. Sally [17] and S. Goto [8] have found that C-M and Buchsbaum local rings of maximal embedding dimension behave well. So we want to extend their results for generalized C-M modules.

Our starting point is the following:

Proposition 4.8. Let $k$ be an infinite field. Then

$$
l(M / \mathfrak{a} N)=e(\mathfrak{a} ; M)+I(M)+d l(M / N)
$$

iff for some or every minimal reduction $a_{1}, \cdots, a_{d}$ of a relative to $N$, the following conditions are satisfied:

(i) $\mathfrak{q} N=\mathfrak{a} N$.

(ii) $a_{1}, \cdots, a_{d}$ is a standard s.o.p. of $M$.

(iii) $\mathfrak{q}_{d-1} M: a_{d} \subseteq N$ by every permutation of $a_{1}, \cdots, a_{d}$.

Proof. $\quad \Rightarrow$ ) By the inequality of Proposition 4.4, we must have $H_{\mathrm{m}}^{0}(M) \subset N$. Hence, by the conditions for equality of Proposition 4.4, we only need to show (ii). Since $a_{1}, \cdots, a_{d}$ is already a standard s.o.p. of $\bar{M}$, by Corollary 2.3 , it suffices to show that $q M \cap H_{\mathrm{m}}^{0}(M)=0$. Note that $a_{1}, \cdots, a_{e}$ are $\left(N / H_{\mathrm{m}}^{0}(M)\right)$-independent by Lemma 4.3. Then we have

$$
\mathfrak{q} M \cap H_{\mathrm{m}}^{0}(M)=\mathfrak{q} N \cap H_{\mathrm{m}}^{0}(M)=0
$$

by Proposition 4.4 (iv).

$(\Leftarrow)$ By Corollary 2.6 (iv), we have

$$
H_{\mathrm{m}}^{0}(M)=0_{M}: a_{d} \subseteq \mathfrak{q}_{e-1} M: a_{d} \subseteq N .
$$


Hence the conclusion follows from Proposition 4.4 by using Corollary 2.3.

Corollary 4.9. Suppose that

$$
l(M / \mathfrak{a} N)=e(\mathfrak{a} ; M)+I(M)+d l(M / N) .
$$

Then the following statements holds:

(i)

$$
\begin{aligned}
l\left(M / \mathfrak{a}^{n} N\right)= & \left(\begin{array}{c}
n+d-1 \\
d
\end{array}\right) e(\mathfrak{a} ; M) \\
& +\sum_{i=1}^{d} \sum_{j=0}^{d-i}\left(\begin{array}{c}
n+d-i-1 \\
d-i
\end{array}\right)\left(\begin{array}{c}
d-i-1 \\
d-1
\end{array}\right) l\left(H_{\mathfrak{m}}^{j}(M)\right) \\
& +\left(\begin{array}{c}
n+d-1 \\
d-1
\end{array}\right) l(M / N)
\end{aligned}
$$

for all $n \geqq 0$.

(ii) a is a standard ideal of $M$ and $N$.

Proof. Without restriction we may assume that $k$ is infinite. Note that $H_{\mathrm{m}}^{0}(M) \subset N$. Then (i) can be easily deduced from Proposition 4.4. Since there exists a generating set $S$ for a such that every $d$ element subset of $S$ forms a minimal reduction of $a$ relative to $N, \mathfrak{a}$ is $M$-standard by Proposition 4.8 (ii) and Proposition 3.2. To show that $\mathfrak{a}$ is $N$-standard, we only need to show that every minimal reduction $a_{1}, \cdots, a_{d}$ of $\mathfrak{a}$ relative to $N$ is a standard s.o.p. of $N$. Note that $\mathfrak{q} N=\mathfrak{a} N$ by Proposition 4.8 (i). Then

$$
\begin{aligned}
I(\mathfrak{q} ; N) & =l(M / \mathfrak{q} N)-l(M / N)-e(\mathfrak{q} ; M) \\
& =l(M / \mathfrak{a} N)-l(M / N)-e(\mathfrak{a} ; M) \\
& =I(M)+(d-1) l(M / N) .
\end{aligned}
$$

To compute $I(N)$ we consider the exact sequence

$$
0 \longrightarrow N \longrightarrow M \longrightarrow M / N \longrightarrow 0 \text {. }
$$

Note that

$$
H_{\mathrm{m}}^{0}(N)=N \cap H_{\mathrm{m}}^{0}(M)=H_{\mathrm{m}}^{0}(M) .
$$

Then from the above exact sequence we get the exact sequence

$$
0 \longrightarrow M / N \longrightarrow H_{\mathrm{m}}^{1}(N) \longrightarrow H_{\mathrm{m}}^{1}(M) \longrightarrow 0 \text {. }
$$

Thus, since $H_{\mathrm{m}}^{i}(N) \cong H_{\mathrm{m}}^{i}(M)$ for $i \geqq 2$, using Lemma 1.5 we get

$$
\begin{aligned}
I(N) & =\sum_{i=0}^{d-1}\left(\begin{array}{c}
d-1 \\
i
\end{array}\right) l\left(H_{\mathfrak{m}}^{i}(N)\right)=\sum_{i=0}^{d-1}\left(\begin{array}{c}
d-1 \\
i
\end{array}\right) l\left(H_{\mathfrak{m}}^{i}(M)\right)+(d-1) l(M / N) \\
& =I(M)+(d-1) l(M / N)=I(\mathfrak{q} ; N) .
\end{aligned}
$$


Hence $a_{1}, \cdots, a_{d}$ is a standard s.o.p. of $N$, as required.

In particular, if

$$
l\left(M / \mathfrak{a}^{2} M\right)=e(\mathfrak{a} ; M)+I(M)+d l(M / \mathfrak{a} M),
$$

Corollary 4.9 (i) gives an explicit formula for $l\left(M / \mathfrak{a}^{n+1} M\right)$ (by replacing $N$ by $a M$ ), which recovers all known results on $\mathrm{H}-\mathrm{S}$ functions of C-M and Buchsbaum local rings of maximal embedding dimension, cf. [8] and [17].

It should be pointed out that there do not exist generalized C-M non-Buchsbaum rings of maximal embedding dimension. This fact follows from the following consequence of Corollary 4.9 (ii):

Corollary 4.10. Suppose that

$$
l(M / \mathfrak{m} N)=e(\mathfrak{m} ; M)+I(M)+d l(M / N) .
$$

Then $M$ and $N$ are Buchsbaum modules.

ExAmple. Let $a_{1}, \cdots, a_{d}$ be a standard s.o.p. of $M$, then

$$
l(M / \mathfrak{a} N)=e(\mathfrak{a} ; M)+I(M)+d l(M / N)
$$

for any module $N \supseteq \mathfrak{q} M+\sum_{i=1}^{d}\left(a_{1}, \cdots, a_{i}, \cdots, a_{d}\right) M: a_{i}$ and any ideal $\mathfrak{a}$ such that $\mathfrak{q} \subseteq \mathfrak{a} \subseteq \mathfrak{q} N: N$. For, we have $\mathfrak{q} N=\mathfrak{a} N$, hence $e(\mathfrak{q} ; N)=e(\mathfrak{a} ; N)$ or, equivalently, $e(\mathfrak{q} ; M)=e(\mathfrak{a} ; M)$ which we really need in the proof of Proposition 4.8 for the above equality instead of the assumption that $a_{1}, \cdots, a_{d}$ is a minimal reduction of a relative to $N$.

\section{§5. Associated graded modules}

In this section, we will study when the associated graded module

$$
G_{\mathrm{a}}(M):=\bigoplus_{n=0}^{\infty} \mathfrak{a}^{n} M / \mathfrak{a}^{n+1} M
$$

of $M$ relative to an ideal $\mathfrak{a}$ of $A$ with $l(M / \mathfrak{a} M)<\infty$ is a graded generalized C-M module over $G_{a}(A)$.

We shall denote by $a^{*}$ the initial form of an element $a$ of $A$ in $G_{a}(A)$, i.e. the image of $a$ in $\mathfrak{a}^{n} / \mathfrak{a}^{n+1}$ where $n$ is the largest integer such that $a \in \mathfrak{a}^{n}$.

We start with the following result which is originally due to S. Goto $[10, \S 3]$ :

Lemma 5.1. Let $a_{1}, \cdots, a_{d}$ be a minimal reduction of a relative to $M$. Let $n_{1}, \cdots, n_{a}$ be arbitrary positive integers. Then 


$$
I\left(a_{1}^{* n_{1}}, \cdots, a_{d}^{* n_{d}} ; G_{a}(M)\right) \geqq I\left(a_{1}^{n_{1}}, \cdots, a_{d}^{n_{d}} ; M\right) .
$$

Equality holds iff

$$
\mathfrak{a}^{n} M \cap\left(a_{1}^{n_{1}}, \cdots, a_{d}^{n_{d}}\right) M=\sum_{i=1}^{d} a_{i}^{n_{i}} \mathfrak{a}^{n-n_{i}} M
$$

for all $n \geqq 0$, where we set $\mathfrak{a}^{m}=A$ if $m<0$.

Proof. We have

$$
\begin{aligned}
& l\left(G_{\mathfrak{a}}(M) /\left(a_{1}^{* n_{1}}, \cdots, a_{d}^{* n_{d}}\right) G_{\mathfrak{a}}(M)\right)=\sum_{n=0}^{\infty} l\left(\mathfrak{a}^{n} M / \sum_{i=1}^{d} a_{i}^{n_{i}} \mathfrak{a}^{n-n_{i}} M+\mathfrak{a}^{n+1} M\right) \\
& \quad \geqq \sum_{n=0}^{\infty} l\left(\mathfrak{a}^{n} M / \mathfrak{a}^{n} M \cap\left(a_{1}^{n_{1}}, \cdots, a_{d}^{n_{d}}\right) M+\mathfrak{a}^{n+1} M\right) \\
& \quad=\sum_{n=0}^{\infty} l\left(\left(a_{1}^{n_{1}}, \cdots, a_{d}^{n_{d}}\right) M+\mathfrak{a}^{n} M /\left(a_{1}^{n_{1}}, \cdots, a_{d}^{n_{d}}\right) M+\mathfrak{a}^{n+1} M\right) \\
& \quad=l\left(M / \bigcap_{n=0}^{\infty}\left(\left(a_{1}^{n_{1}}, \cdots, a_{d}^{n_{d}}\right) M+\mathfrak{a}^{n+1} M\right)\right)=l\left(M /\left(a_{1}^{n_{1}}, \cdots, a_{d}^{n_{d}}\right) M\right) .
\end{aligned}
$$

Note that

$$
\begin{gathered}
e\left(a_{1}^{* n_{1}}, \cdots, a_{d}^{* n_{d}} ; G_{\mathrm{a}}(M)\right)=n_{1} \cdots n_{d} e\left(a_{1}^{*}, \cdots, a_{d}^{*} ; G_{a}(M)\right) \\
=n_{1} \cdots n_{d} e(\mathfrak{a} ; M)=e\left(a_{1}^{n_{1}}, \cdots, \mathfrak{a}_{d}^{n_{d}} ; M\right) .
\end{gathered}
$$

Then we get

$$
I\left(a_{1}^{* n_{1}}, \cdots, a_{d}^{* n_{d}} ; G_{a}(M)\right) \geqq I\left(a_{1}^{n_{1}}, \cdots, a_{d}^{n_{d}} ; M\right),
$$

which will be an equality if

$$
\mathfrak{a}^{n} M \cap\left(a_{1}^{n_{1}}, \cdots, a_{d}^{n_{d}}\right) M=\sum_{i=1}^{d} a_{i}^{n_{i}} \mathfrak{a}^{n-n_{i}} M .
$$

Conversely, assume that (1) is an equality. Then we must have

$$
\mathfrak{a}^{n} M \cap\left(a_{1}^{n_{1}}, \cdots, a_{d}^{n_{d}}\right) M \subseteq \sum_{i=1}^{d} a_{i}^{n_{i}} \mathfrak{a}^{n_{-n_{i}}} M+\mathfrak{a}^{n+1} M
$$

for all $n \geqq 0$. Hence

$$
\begin{gathered}
\mathfrak{a}^{n} M \cap\left(a_{1}^{n_{1}}, \cdots, a_{d}^{n_{d}}\right) M=\sum_{i=1}^{d} a_{i}^{n_{i}} \mathfrak{a}^{n-n_{i}} M+\mathfrak{a}^{n+1} M \cap\left(a_{1}^{n_{1}}, \cdots, a_{d}^{n_{d}}\right) M \\
\subseteq \sum_{i=1}^{d} a_{i}^{n_{i}} \mathfrak{a}^{n-n_{i}} M+\mathfrak{a}^{n+2} M \subseteq \cdots \subseteq \\
\subseteq \bigcap_{m=n+1}^{\infty}\left(\sum_{i=1}^{d} a_{i}^{n_{i}} \mathfrak{a}^{n^{n-n_{i}}} M+\mathfrak{a}^{m} M\right)=\sum_{i=1}^{d} a_{i}^{n_{i}} \mathfrak{a}^{n-n i} M,
\end{gathered}
$$

which then implies (2), as required. 
From Lemma 5.1 we can conclude that the property of being a generalized C-M module is transferred from $G_{a}(M)$ to $M$.

Corollary 5.2 [10, Proposition 3.1]. Suppose that $G_{a}(M)$ is a generalized $C$ - $M$ module. Then so is $M$ with $I(M) \leqq I\left(G_{a}(M)\right)$.

Proof. Without restriction we may assume that $k$ is an infinite field. Then we can find a minimal reduction $a_{1}, \cdots, \mathfrak{a}_{d}$ of a relative to $M$. By Lemma 5.1, we have

$$
I\left(a_{1}^{n_{1}}, \cdots, a_{d}^{n_{d}} ; M\right) \leqq I\left(G_{\mathrm{a}}(M)\right)
$$

for all positive integers $n_{1}, \cdots, n_{d}$. Hence $M$ is a generalized C-M module with $I(M) \leqq I\left(G_{\mathrm{a}}(M)\right.$ ) by Lemma 1.1 (iii) and Lemma 1.5 .

In general, it is hard to find conditions for the converse of Corollary 5.2. We can only prove this converse if $a$ is generated by a standard s.o.p. of $M$ or if $l\left(M / \mathfrak{a}^{2} M\right)$ attains some extreme value.

First, we will exhibit some properties of $d$-sequences (hence of standard s.o.p.'s) related to this topic.

Lemma 5.3 (see e.g. $[27, \S 3]$ ). Let $a_{1}, \cdots, a_{d}$ be a $d$-sequence of $M$. Then

(i) $G_{\mathrm{q}}(M) /\left(a_{1}^{*}, \cdots, a_{i}^{*}\right) G_{\mathrm{q}}(M) \cong G_{\mathrm{q}}\left(M / \mathfrak{q}_{i} M\right), i=1, \cdots, d-1$.

(ii) $G_{\mathrm{q}}(M) \cong S_{\mathrm{q}}(M) / q S_{\mathrm{q}}(M)$, where $S_{\mathrm{q}}(M)$ denotes the symmetric module of $M$ with respect to $\mathfrak{q}$.

Now we are ready to investigate the associated graded module $G_{q}(M)$ of a standard parameter ideal $\mathfrak{q}$ of $M$. We will denote by $P$ the maximal graded ideal of $G_{\mathrm{q}}(A)$.

TheOREM 5.4 (cf. [4, (10.1)] and [9, Theorem 1.1]). Let $a_{1}, \cdots, a_{d}$ be a standard s.o.p. of $M$. Then $G_{q}(M)$ is a generalized $C$ - $M$ module with

$$
H_{P}^{i}\left(G_{\mathrm{q}}(M)\right)=H_{\mathrm{m}}^{i}(M)(i)
$$

for $i=0, \cdots, d-1$, where $H_{\mathrm{u}}^{i}(M)$ is considered as a graded module concentrated in degree 0 (the integer in the round brackets denotes the shifting degree), and $\left[H_{P}^{d}\left(G_{\uparrow}(M)\right)\right]_{n}=0$ for $n>-d$.

Proof. Note that $\mathfrak{q} M \cap H_{\mathrm{m}}^{0}(M)=0$ by Corollary 2.3. Then

$$
\begin{aligned}
{\left[G_{\mathfrak{q}}(\bar{M})\right]_{n} } & =\mathfrak{q}^{n} M+H_{\mathrm{m}}^{0}(M) / \mathfrak{q}^{n+1} M+H_{\mathrm{m}}^{0}(M) \cong \mathfrak{q}^{n} M / \mathfrak{q}^{n} M \cap H_{\mathrm{m}}^{0}(M)+\mathfrak{q}^{n+1} M \\
& =\mathfrak{q}^{n} M / \mathfrak{q}^{n+1} M=\left[G_{\mathfrak{q}}(M)\right]_{n}
\end{aligned}
$$


for all $n>0$. Hence we have the exact sequence

$$
0 \longrightarrow H_{\mathrm{m}}^{0}(M) \longrightarrow G_{\mathrm{q}}(M) \longrightarrow G_{\mathrm{q}}(\bar{M}) \longrightarrow 0 \text {. }
$$

Now, it is clear that we only need to prove the statement for $\bar{M}$, i.e. we may assume that depth $M>0$. In this case, using Corollary 2.6 (v), we have

$$
\left[0_{G_{q}(M)}: a_{1}^{*}\right]_{n}=\mathfrak{q}^{n} M \cap\left(\mathfrak{q}^{n+2} M ; a_{1}\right) / \mathfrak{q}^{n+1} M=0
$$

for all $n \geqq 0$. Therefore, $a_{1}^{*}$ is a non-zerodivisor of $G_{4}(M)$. Hence $H_{P}^{0}\left(G_{\mathrm{q}}(M)\right)=0$. Moreover, we have the exact sequence

$$
0 \longrightarrow G_{\mathrm{q}}(M) \stackrel{a_{1}^{*}}{\longrightarrow} G_{\mathrm{q}}(M) \longrightarrow G_{\mathrm{q}}(M) / a_{1}^{*} G_{\mathrm{q}}(M) \longrightarrow 0 .
$$

Note that by Lemma 5.2 (i),

$$
G_{\mathrm{q}}(M) / a_{1}^{*} G_{\mathrm{q}}(M) \cong G_{\mathrm{q}}\left(M / a_{1} M\right) .
$$

If $d=1, G_{\mathrm{a}}\left(M / a_{1} M\right)=M / a_{1} M$. Hence from (2) we get

$$
\left[H_{P}^{1}\left(G_{\mathrm{q}}(M)\right)\right]_{n} \stackrel{a_{1}^{*}}{\longrightarrow}\left[H_{P}^{1}\left(G_{\mathrm{q}}(M)\right)\right]_{n+1}
$$

for all $n \geqq 0$. Since every element of $H_{P}^{1}\left(G_{\mathrm{q}}(M)\right)$ is annihilated by some power of $a_{1}^{*}$, we must have $\left[H_{P}^{1}\left(G_{q}(M)\right)\right]_{n}=0$ for all $n \geqq 0$. So we have proved the case $d=1$. If $d>1$, by induction we may assume that

$$
H_{P}^{i}\left(G_{\mathrm{q}}\left(M / a_{1} M\right)\right)=H_{\mathrm{m}}^{i}\left(M / a_{1} M\right)(i)
$$

for $i=0, \cdots, d-2$, and $\left[H_{P}^{d-1}\left(G_{\mathrm{q}}\left(M / a_{1} M\right)\right)\right]_{n}=0$ for $n>1-d$. Then from (2) we can deduce that

$$
\left[H_{P}^{i}\left(G_{q}(M)\right)\right]_{n} \stackrel{a_{1}^{*}}{\longrightarrow}\left[H_{P}^{i}\left(G_{q}(M)\right)\right]_{n+1}
$$

and, therefore, $\left[H_{P}^{i}\left(G_{q}(M)\right)\right]_{n}=0$ for all $n>-i, i=1, \cdots, d$. For $n=$ $-i, i=1, \cdots, d-1$, we may assume, by induction, that there exist isomorphisms $\delta_{i-1}$ and $\varepsilon_{i-1}$ which make the following diagram commutative:

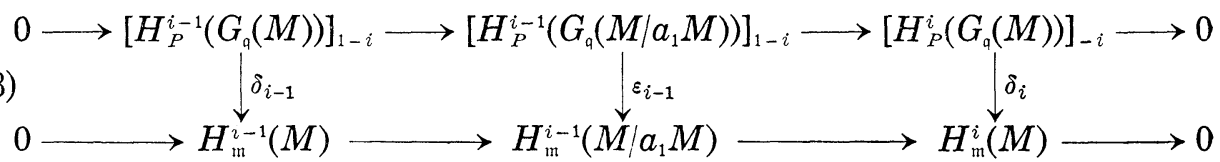

where the short exactness of the lower sequence follows from the fact $a_{1} H_{\mathrm{m}}^{i-1}(M)=a_{1} H_{\mathrm{m}}^{i}(M)=0$ of Theorem 2.5, and $\delta_{i}$ is the induced isomor- 
phism. It remains to show, for the induction proof, that the diagram

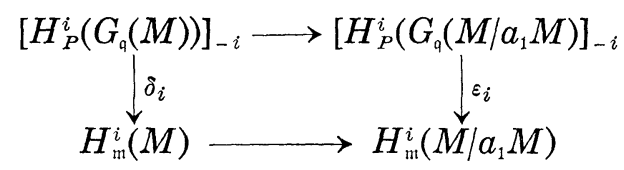

is commutative. For that we consider the following cubic diagram:

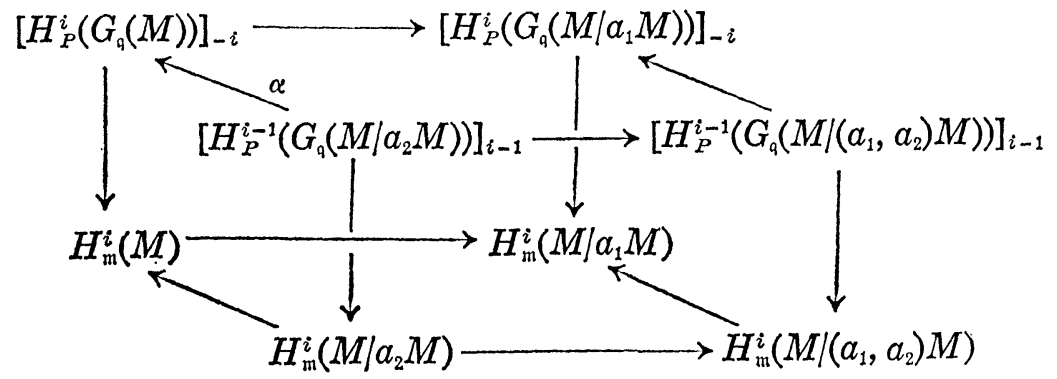

where the top face is derived from the commutative diagram

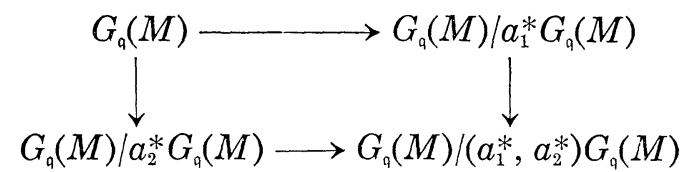

by using Lemma 5.3 (i). The commutativity of the left face has been shown in (3) by replacing $a_{1}$ by $a_{2}$. By induction we may also assume that the front and right faces are commutative (the assumption depth $M>0$ does not cause us any problem because (1)). That the bottom face is commutative is immediate. Thus, since $\alpha$ is surjective by virtue of (3), we can conclude that the back face, i.e. (4), is commutative too. So we have proved that

$$
\left[H_{P}^{i}\left(G_{q}(M)\right)\right]_{-i} \cong H_{\mathrm{m}}^{i}(M) .
$$

For $n=1-i$, we consider the commutative diagram

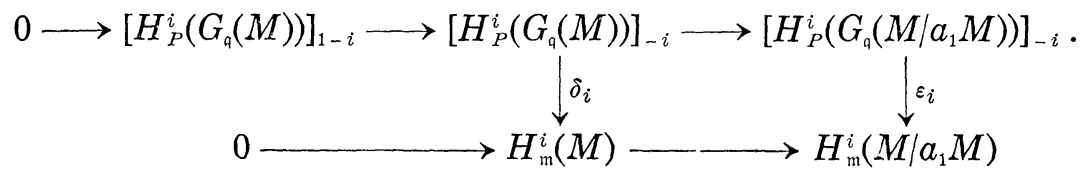

Then we can see that $\left[H_{P}^{i}\left(G_{\mathrm{q}}(M)\right)\right]_{1-i}=0$. For $n<1-\mathrm{i}$, we have

$$
\left[H_{P}^{i}\left(G_{q}(M)\right)\right]_{n} \cong\left[H_{P}^{i}\left(G_{q}(M)\right)\right]_{n+1}
$$


by (2) and the induction hypothesis on $M / a_{1} M$. Hence

$$
\left[H_{P}^{i}\left(G_{\mathrm{q}}(M)\right)\right]_{n} \cong\left[H_{P}^{i}\left(G_{\mathrm{q}}(M)\right)\right]_{1-i}=0,
$$

as required. The proof of Theorem 5.4 is now complete.

Theorem 5.4 has some interesting consequences. First, we can show that the property of being a standard s.o.p. is reserved between $M$ and $G_{\mathrm{a}}(M)$ in the following sense:

Corollary 5.5. $a_{1}, \cdots, a_{d}$ is a standard s.o.p. of $M$ iff $a_{1}^{*}, \cdots, a_{d}^{*}$ is a standard s.o.p. of $G_{\mathrm{q}}(M)$.

Proof. $\quad \Leftrightarrow$ ) follows from Corollary 3.12 and Theorem 5.4. For $(\Leftarrow)$ we know, by Corollary 5.2 , that $M$ is a generalized C-M module with

$$
I(M) \leqq I\left(G_{\mathfrak{q}}(M)\right)=I\left(a_{1}^{*}, \cdots, a_{d}^{*} ; G_{\mathfrak{q}}(M)\right)=I(\mathfrak{q} ; M) \leqq I(M) .
$$

Therefore, we must have $I(\mathfrak{q} ; M)=I(M)$.

Moreover, we have the following property of powers of standard parameter ideals:

Corollary 5.6 (cf. [9, Corollary 1.2]). Let $a_{1}, \cdots, a_{d}$ be a standard s.o.p. of $M$. Then

$$
\left(a_{1}^{n_{1}}, \cdots, a_{d}^{n_{d}}\right) M \cap \mathfrak{q}^{n} M=\sum_{i=1}^{d} a_{i}^{n_{i}} \mathfrak{q}^{n-n_{i}} M
$$

for all positive integers $n_{1}, \cdots, n_{d}$ and $n \geqq 0$.

Proof. By Lemma 1.5 and Theorem 5.4, we have $I\left(G_{\mathrm{q}}(M)\right)=I(M)$. By Corollary 3.3 and Corollary 5.5, $a_{1}^{* n_{1}}, \cdots, a_{d}^{* n_{d}}$ and $a_{1}^{n_{1}}, \cdots, a_{d}^{n_{d}}$ are standard s.o.p.'s $G_{q}(M)$ and $M$, respectively. Hence

$$
I\left(a_{1}^{* n_{1}}, \cdots, a_{d}^{* n_{d}} ; G_{\mathrm{q}}(M)\right)=I\left(a_{1}^{n_{1}}, \cdots, a_{d}^{n_{d}} ; M\right),
$$

which then implies the statement by Lemma 5.1 .

Now, we will give a criterion for an irrelevant graded ideal of $G_{\mathrm{q}}(M)$ to be $G_{\mathrm{q}}(M)$-standard.

Theorem 5.7. Let $a_{1}, \cdots, a_{d}$ be an arbitrary s.o.p. of $M$. Let $\mathfrak{a} \supseteq \mathfrak{q}$ be an ideal of $A$. Let $\mathfrak{a}^{*}$ denote the ideal of $G_{\mathrm{q}}(A)$ generated by the initial forms of the elements of $a$. Then $a^{*}$ is $G_{a}(M)$-standard iff the following conditions are satisfied:

(i) $a_{1}, \cdots, a_{d}$ is a standard s.o.p. of $M$.

(ii) $\mathfrak{q}_{d-1} M: a_{d} \subseteq \mathfrak{q}_{d-1} M: \mathfrak{a}$ by every permutation of $a_{1}, \cdots, a_{d}$. 
Proof. $(\Rightarrow)$ (i) follows from Corollary 5.5. For (ii) it suffices to show that $a H_{\mathrm{m}}^{0}\left(M / \mathfrak{q}_{d-1} M\right)=0$. Since $a_{d}$ is a standard s.o.p. of $M / \mathfrak{q}_{d-1} M$ by Corollary 2.4, we have

$$
H_{\mathrm{m}}^{0}\left(M / \mathfrak{q}_{d-1} M\right)=H_{P}^{0}\left(G_{q}\left(M / \mathfrak{q}_{d-1} M\right)\right)
$$

by Theorem 5.4. But

$$
G_{\mathrm{q}}\left(M / \mathfrak{q}_{d-1} M\right) \cong G_{\mathrm{q}}(M) /\left(a_{1}^{*}, \cdots, a_{d-1}^{*}\right) G_{\mathrm{q}}(M)
$$

by Lemma 5.3 (i). Hence, from the fact that

$$
\mathfrak{a}^{*} H_{P}^{0}\left(G_{\mathrm{q}}(M) /\left(a_{1}^{*}, \cdots, a_{d-1}^{*}\right) G_{\mathrm{q}}(M)=0\right.
$$

$\left(a_{1}^{*}, \cdots, a_{d}^{*}\right.$ is a $a^{*}$-weak $G_{q}(M)$-sequence by Proposition 3.1), we can easily conclude that $a H_{\mathrm{m}}^{0}\left(M / \mathfrak{q}_{d-1} M\right)=0$.

$(\Leftarrow)$ From (i) and Theorem 5.4 we get $H_{P}^{0}\left(G_{\mathrm{q}}(M)\right) \cong H_{\mathrm{m}}^{0}(M)$, where $H_{\mathrm{m}}^{0}(M)$ is considered as a graded module concentrated in degree 0 . Thus, if $d=1$, we have $\mathfrak{a}^{*} H_{P}^{0}\left(G_{\mathrm{q}}(M)\right)=0$ because $a H_{\mathrm{m}}^{0}(M)=0$ by (ii). Hence $a^{*}$ is $G_{a}(M)$-standard by Theorem 2.4. For $d>1$, we first consider the case depth $M>0$.

In this case, $a_{1}^{*}$ is a non-zerodivisor of $G_{\mathrm{a}}(M)$. Further, by induction, we may assume that $\mathfrak{a}^{*}$ is $G_{\mathfrak{q}}\left(M / a_{1} M\right)$-standard. Note that

$$
G_{\mathrm{q}}\left(M / a_{1} M\right) \cong G_{\mathrm{q}}(M) / a_{1}^{*} G_{\mathrm{q}}(M)
$$

by Lemma 5.3 (i) and that

$$
a_{1}^{*} H_{P}^{i}\left(G_{\mathrm{q}}(M)\right)=0
$$

by Theorem 5.4. Then $\mathfrak{a}^{*}$ is $G_{q}(M)$-standard by Corollary 3.8 .

For the case depth $M=0$, we first note that

$$
G_{\mathrm{q}}(M) / H_{P}^{0}\left(G_{\mathrm{q}}(M)\right) \cong G_{\mathrm{q}}(\bar{M})
$$

by the proof of Theorem 5.4. By Corollary 2.3, $a_{1}, \cdots, a_{d}$ is still a standard s.o.p. of $\bar{M}$. Moreover, using Corollary 2.6 (iv), we also have

$$
\begin{aligned}
\left(\mathfrak{q}_{d-1} M+H_{\mathrm{m}}^{0}(M)\right): a_{d} & \subseteq \bigcup_{n=1}^{\infty} \mathfrak{q}_{d-1} M: m^{n}=\mathfrak{q}_{d-1} M: a_{d} \subseteq q_{d-1} M: \mathfrak{a} \\
& \subseteq\left(q_{d-1} M+H_{\mathrm{m}}^{0}(M)\right): \mathfrak{a} .
\end{aligned}
$$

Hence $\mathfrak{a}^{*}$ is $G_{\mathrm{q}}(\bar{M})$-standard by the above proof for the case depth $M<0$. Now, by Corollary 3.9 , it remains to find a $G_{q}(M)$-base $S$ for $\mathfrak{a}^{*}$ such that for every $d$ element subset $\left\{f_{1}, \cdots, f_{d}\right\}$ of $S$, 


$$
\left(f_{1}, \cdots, f_{d}\right) G_{\mathrm{q}}(M) \cap H_{P}^{0}\left(G_{\mathrm{q}}(M)\right)=0 .
$$

Of course, we may assume that the residue field $k$ is infinite. Since $\mathfrak{a}^{*} / P \mathfrak{a}^{*}=\mathfrak{a} / \mathfrak{m a} \oplus \mathfrak{q} / \mathfrak{m q}$, we can find a $G_{\mathfrak{a}}(M)$-base $S$ for $\mathfrak{a}^{*}$ such that every element $f \in S$ has the form $b^{*}+c^{*}$ for some $b \in \mathfrak{a}, c \in \mathfrak{q} \mid \mathrm{mq}$. Let $b_{1}^{*}+c_{1}^{*}$, $\cdots, b_{d}^{*}+c_{d}^{*}$ be $d$ elements of $S$. Note that

$$
\left(b_{i}^{*}+c_{i}^{*}\right)^{n} \in c_{i}^{*} G_{\mathrm{q}}(M)
$$

for all $i=1, \cdots, d, n$ large. Then $c_{1}^{*}, \cdots, c_{d}^{*}$ also form a s.o.p. of $G_{q}(M)$. From this it follows that

$$
\left(c_{1}^{*}, \cdots, c_{d}^{*}\right) G_{\mathrm{q}}(A)=\left(a_{1}^{*}, \cdots, a_{d}^{*}\right) G_{\mathrm{q}}(M) .
$$

Hence we can write

$$
c_{i}=\sum_{j=1}^{d} c_{i j} a_{\jmath}+d_{i}
$$

for some units $c_{i j}$ of $A$ and $d_{i} \in \mathfrak{m} q, i=1, \cdots, d$, such that $\operatorname{det}\left(a_{i j}\right)$ is a unit of $A$. Now, by Lemma 5.3 (ii), we will represent $G_{q}(M)$ as $S_{q}(M) / q S_{q}(M)$. If we denote by $A[X]$ the polynomial ring over $A$ in $d$ indeterminates $X_{1}, \cdots, X_{d}$, then $S_{q}(M)$ is defined to be the factor of $M[X]=M \otimes_{A} A[X]$ by the submodule $F$ generated by all elements $m_{1} X_{1}+\cdots+m_{d} X_{d}$ such that $a_{1} m_{1}+\cdots+a_{d} m_{d}=0$. Hence

$$
G_{\mathrm{q}}(M)=M[X] / \mathfrak{q} M[X]+F .
$$

Put

$$
Y_{i}=\sum_{j=1}^{d} c_{i j} X_{j}+d_{i}+b_{i},
$$

$i=1, \cdots, d$. Then $A[X]=A\left[Y_{1}, \cdots, Y_{d}\right]$. Further, using (ii) we can easily verify that

$$
F \subseteq\left(Y_{1}, \cdots, Y_{d}, \mathfrak{q}\right) M[X]
$$

Let $g$ be an arbitrary element of $\left(b_{1}^{*}+c_{1}^{*}, \cdots, b_{d}^{*}+c_{d}^{*}\right) G_{q}(M) \cap H_{P}^{0}\left(G_{q}(M)\right)$. Note that $H_{P}^{0}\left(G_{\mathrm{q}}(M)\right) \cong H_{\mathrm{m}}^{0}(M)$, Then we can find a representative $h$ of $g$ in $\left(Y_{1}, \cdots, Y_{d}, \mathfrak{q}\right) M[X] \cap H_{\mathrm{m}}^{0}(M)$. Since $Y_{1}, \cdots, Y_{d}$ may be considered as indeterminates over $A$, we must have $h \in \mathfrak{q} M \cap H_{\mathrm{m}}^{0}(M)=0$ by Corollary 2.3. Hence $g=0$, as required. The proof of Theorem 5.7 is now complete.

Remark 5.8. From the above proof one can easily verify that the conditions of Theorem 5.7 may be replaced by the condition 


$$
\mathfrak{a} H_{\mathrm{m}}^{i}\left(M / \mathfrak{q}_{j} M\right)=0
$$

for all non-negative integers $i, j$ with $i+j<d$ and every permutation of $a_{1}, \cdots, a_{d}$. In particular, that is always satisfied if $\mathfrak{a}$ is a standard ideal of $M$.

Concerning the theory of Buchsbaum modules we have the following interesting consequences:

Corollary 5.9. $M$ is a Buchsbaum module iff so is $G_{q}(M)$ for every parameter ideal $\mathfrak{q}$ of $M$.

Proof. Straightforward.

Corollary 5.10. $M$ is a quasi-Buchsbaum module, i.e. $\mathrm{m}_{\mathrm{m}}^{i}(M)=0$ for $i=0, \cdots, d-1$, iff $G_{q}(M)$ is a Buchsbaum module for some parameter ideal $\mathfrak{q}$ of $M$.

Proof. $(\Rightarrow)$ follows from Theorem 5.7 and the fact that every s.o.p. of $M$ contained in large powers of $\mathfrak{m}$ is standard and $m$-weak [20]. For $(\Leftarrow)$ we first note that $a_{1}, \cdots, a_{d}$ is a standard s.o.p. of $M$ by Corollary 5.5. Thus, we can apply Theorem 5.4 to show that $\mathrm{m}_{\mathrm{m}}^{i}(M)=0$ by using the fact $P H_{P}^{i}\left(G_{\mathrm{q}}(M)\right)=0, i=0, \cdots, d-1$.

Now we will study $G_{a}(M)$ in the case $l\left(M / \mathfrak{a}^{2} M\right)$ attains some extreme value (the module-version of Buchsbaum rings with maximal embedding dimension).

Proposition 5.11 (cf. [8, Theorem 1.1]). Suppose that $M$ is a generalized $C-M$ module and

$$
l\left(M / \mathfrak{a}^{2} M\right)=e(\mathfrak{a} ; M)+I(M)+d l(M / \mathfrak{a} M) .
$$

Then $G_{a}(M)$ is a generalized $C-M$ module with

$$
H_{Q}^{i}\left(G_{\mathrm{a}}(M)\right) \cong H_{\mathrm{m}}^{i}(M)(i-1)
$$

for $i=0, \cdots, d-1$, and $\left[H_{Q}^{d}\left(G_{\mathrm{a}}(M)\right)\right]_{n}=0$ for $n>1-d$, where $Q$ denotes the maximal graded ideal of $G_{a}(A)$. Moreover, the ideal $a^{*}$ of $G_{a}(A)$ generated by the initial forms of the elements of $a$ is $G_{a}(M)$-standard.

Proof. Without restriction we may assume that the residue field $k$ is infinite. Let $a_{1}, \cdots, a_{d}$ be a minimal reduction of a relative to $a M$. Then $q \mathfrak{a} M=\mathfrak{a}^{2} M$ by Proposition 4.8 (i). Hence we have the following exact sequence of $G_{\mathrm{q}}(A)$-modules: 


$$
0 \longrightarrow \sum_{t=0}^{\infty} \mathfrak{q}^{t} M / \mathfrak{q}^{t} \mathfrak{a} M \longrightarrow \bigoplus_{t=0}^{\infty} \mathfrak{a}^{t} M / \mathfrak{a}^{t+1} M \longrightarrow\left(\bigoplus_{t=0}^{\infty} \mathfrak{q}^{t} \mathfrak{a} M / \mathfrak{q}^{t+1} M\right)(-1) \longrightarrow 0 .
$$

By Lemma 4.3, from Proposition 4.8 (ii) and (iii) we get

$$
\bigoplus_{t=0}^{\infty} \mathfrak{q}^{t} M / \mathfrak{q}^{t} \mathfrak{a} M \cong(M / \mathfrak{a} M)\left[X_{1}, \cdots, X_{d}\right]
$$

which is a C-M module over $G_{\mathrm{q}}(A)$ whose $d$ th local cohomology module concentrated in degree $\leqq-d$. Thus, from the above exact sequence we can deduce that

$$
H_{P}^{i}\left(G_{a}(M)\right) \cong\left(H_{P}^{i}\left(\bigoplus_{t=0}^{\infty} \mathfrak{q}^{t} \mathfrak{a} M / \mathfrak{q}^{t+1} M\right)\right)(-1)
$$

for $i=0, \cdots, d-1$, and

$$
\left[H_{P}^{d}\left(G_{\mathrm{a}}(M)\right)\right]_{n} \cong\left[H_{P}^{d}\left(\bigoplus_{t=0}^{\infty} \mathfrak{q}^{t} \mathfrak{a} M / \mathfrak{q}^{t+1} M\right)\right]_{n+1}
$$

for $n>-d$. On the other hand, we also have the exact sequence

$$
0 \longrightarrow \bigoplus_{t=0} \mathfrak{q}^{t} \mathfrak{a} M / \mathfrak{q}^{t+1} M \longrightarrow \bigoplus_{t=0} \mathfrak{q}^{t} M / \mathfrak{q}^{t+1} M \longrightarrow \bigoplus_{t=0} \mathfrak{q}^{t} M / \mathfrak{q}^{t} \mathfrak{a} M \longrightarrow 0
$$

From this it follows that

$$
H_{P}^{i}\left(\bigoplus_{t=0}^{\infty} \mathfrak{q}^{t} \mathfrak{a} M / \mathfrak{q}^{t+1} M\right) \cong H_{P}^{i}\left(G_{\mathfrak{q}}(M)\right)
$$

for $i=0, \cdots, d-1$, and

$$
\left[H_{P}^{d}\left(\bigoplus_{t=0}^{\infty} \mathfrak{q}^{t} \mathfrak{a} M / \mathfrak{q}^{t+1} M\right)\right]_{n} \cong\left[H_{P}^{d}\left(G_{q}(M)\right)\right]_{n}
$$

for $n>-d$. But by Proposition 4.8 (ii), $a_{1}, \cdots, a_{d}$ is a standard s.o.p. of $M$. Hence we can estimate the local cohomology modules of $G_{\mathrm{q}}(M)$ by Theorem 5.4. Note that

$$
H_{Q}^{i}\left(G_{a}(M)\right)=\underline{\lim }_{t} H^{i}\left(a_{1}^{* t}, \cdots, a_{d}^{* t} ; G_{a}(M)\right)=H_{P}^{i}\left(G_{\mathrm{a}}(M)\right),
$$

$i=0, \cdots, d$, where $a_{1}^{*}, \cdots, a_{d}^{*}$ may be understood both as elements of $G_{\mathrm{a}}(A)$ and $G_{\mathrm{a}}(A)$. Then from the above relations we can easily derive the formulas for $H_{Q}^{i}\left(G_{a}(M)\right)$ as required. The fact that $\mathfrak{a}^{*}$ is $G_{a}(M)$ standard follows from Corollary 3.12.

Similar to Corollary 5.6 of Theorem 5.4, we have the following consequence: 
Corollary 5.12. Let $M$ and $a$ be as in Proposition 5.11. Let $a_{1}, \cdots$, $a_{d}$ be a minimal reduction of a relative to $M$. Then

$$
\left(a_{1}^{n_{1}}, \cdots, a_{d}^{n_{d}}\right) M \cap \mathfrak{a}^{n} M=\sum_{i=1}^{d} a_{i}^{n_{i}} \mathfrak{a}^{n-n_{i}} M
$$

for all positive integers $n_{1}, \cdots, n_{d}$ and $n \geqq 0$.

\section{§6. Rees modules}

Let $\mathfrak{a}$ be an ideal of $A$ with $l(M / \mathfrak{a} M)<\infty$. Then we call the graded module

$$
R_{\mathrm{a}}(M):=\bigoplus_{n=0}^{\infty} \mathfrak{a}^{n} M
$$

over the Rees algebra $R_{\mathrm{a}}(A)$ the Rees module of $M$ relative to a. It is also known under the name arithmetical blowing-up [3], [4].

It is well-known that $G_{a}(M)=R_{a}(M) / a R_{a}(M)$ and that $a R_{a}(M)$ may be identified with the positively graded part of $R_{a}(M)$ by an isomorphism of degree -1 . From these facts we can show that concerning the property of being a generalized C-M module, there is a close relationship between $G_{a}(M)$ and $R_{\mathrm{a}}(M)$.

We shall denote by $Q$ the maximal graded ideal of $G_{a}(A)$.

Proposition 6.1. The following conditions are equivalent:

(i) $M$ and $R_{\mathrm{a}}(M)$ are generalized $C$ - $M$ modules.

(ii) $G_{a}(M)$ is a generalized $C-M$ module.

Proof. (i) $\Rightarrow$ (ii). From the exact sequences

$$
\begin{aligned}
& 0 \longrightarrow a R_{\mathrm{a}}(M)(-1) \longrightarrow R_{\mathrm{a}}(M) \longrightarrow M \longrightarrow 0 \\
& 0 \longrightarrow a R_{\mathrm{a}}(M) \longrightarrow R_{\mathrm{a}}(M) \longrightarrow G_{\mathrm{a}}(M) \longrightarrow 0
\end{aligned}
$$

we can easily deduce that first, $a R_{a}(M)$ and then $G_{a}(M)$ is a generalized C-M module.

(ii) $\Rightarrow$ (i). That $M$ is a generalized C-M module follows from Corollary 5.2. To show that $R_{\mathrm{a}}(M)$ is a generalized C-M module, we may assume, without loss of generality, that $A$ is a complete local ring. Then $A$ is a factor of a regular ring, hence so is $R_{\mathrm{a}}(A)$. By Lemma 1.2 (iv) and Lemma 1.4, it suffices to show:

(1) $\operatorname{dim} R_{\mathrm{a}}(A) / P=d+1$ for any minimal prime divisor $P$ of $R_{\mathrm{a}}(M)$.

(2) $\left[R_{a}(M)\right]_{P}$ is a C-M module for every prime ideal $p \neq Q$ of $\operatorname{Supp}\left(R_{\mathrm{a}}(M)\right)$. 
For (1) we identify $R_{\mathrm{a}}(A)$ with the subring $A[\mathfrak{a} T]$ of $A[T]$ ( $T$ is an indeterminate) generated over $A$ by all elements $a T, a \in \mathfrak{a}$. It is easy to see that

$$
\operatorname{Ann}\left(R_{\mathrm{a}}(M)\right)=\operatorname{Ann}(M) A[T] \cap A[\mathfrak{a} T],
$$

where Ann denotes the annihilator. From this it follows that

$$
R_{\mathrm{a}}(A) / \operatorname{Ann}\left(R_{\mathrm{a}}(M)\right)=R_{\mathrm{a}}(A / \operatorname{Ann}(M)) .
$$

Therefore, since $\operatorname{dim} A / \mathfrak{p}=d$ for any minimal prime ideal over Ann $(M)$ by Lemma 1.2 (iv), $\operatorname{dim} R_{\mathrm{a}}(A) / P=d+1$ for any minimal prime ideal $P$ over Ann $\left(R_{\mathrm{a}}(M)\right)$ by $[28, \S 1]$.

For (2) we set $\mathfrak{p}=P \cap A$. If $\mathfrak{a} \not \subset \mathfrak{p}$, we have $[A[\mathfrak{a} T]]_{\mathfrak{p}}=A_{\mathfrak{p}}[T]$. Hence $\left[R_{\mathrm{a}}(M)\right]_{\mathfrak{p}}=M_{\mathfrak{p}}[T]$. Since by Lemma 1.2 (iv), $M_{\mathfrak{p}}$ is a C-M module, so is $\left[R_{\mathrm{a}}(M)\right]_{P}$ as a localization of $\left[R_{\mathfrak{a}}(M)\right]_{\mathfrak{p}}$. If $\mathfrak{a} \subseteq \mathfrak{p}$, i.e. $\mathfrak{p}=\mathfrak{m}$, there exists some element $a \in \mathfrak{a}$ such that $a T \notin P$ because $P \neq Q=(\mathfrak{m}, \mathfrak{a} T) A[\mathfrak{a} T]$. Since $\mathfrak{a} \subset a A\left[\mathfrak{a} T,(a T)^{-1}\right]$,

$$
\left[R_{\mathrm{a}}(M)\right] / a\left[R_{\mathrm{a}}(M)\right]_{P}=\left[R_{\mathrm{a}}(M)\right]_{P} / \mathfrak{a}\left[R_{\mathrm{a}}(M)\right]_{P}=\left[G_{\mathrm{a}}(M)\right]_{P},
$$

which is a C-M module by Lemma 1.2 (iv). Moreover, since $0_{M}$ is unmixed up to $\mathfrak{m}$ by Lemma 1.2 (iii), using a module-version of [28, Proposition 1.1 (iii)], we can show that $a$ is a non-zerodivisor of $\left[R_{\mathrm{a}}(M)\right]_{P}$. Hence $\left[R_{\mathrm{a}}(M)\right]_{P}$. is a C-M module, as required. The proof of Proposition 6.1 is now complete.

Now, we want to study $R_{a}(M)$ in the case $\mathfrak{a}=\mathfrak{q}$ is a standard parameter ideal. Note that in this case, $R_{\mathrm{q}}(M)=S_{\mathrm{q}}(M)$, which follows from property of $d$-sequences, see [13], [14], [27], [29]. Then the following result also holds for symmetric modules:

THEOREM 6.2 (cf. [4, (11.1)]). Let $a_{1}, \cdots, a_{d}$ be a standard s.o.p. of $M$. Then $R_{\mathrm{q}}(M)$ is a generalized $C$ - $M$ module with

$$
\begin{gathered}
H_{Q}^{0}\left(R_{\mathrm{q}}(M)\right)=H_{\mathrm{m}}^{0}(M) \\
H_{Q}^{i}\left(R_{\mathrm{q}}(M)\right)=\underset{i-2 \geqq n \geqq 1}{\bigoplus} H_{\mathfrak{m}}^{i-1}(M)(n)
\end{gathered}
$$

for $i=1, \cdots, d$, where $H_{\mathrm{m}}^{i}(M)$ is considered as a graded module over $R_{\mathrm{q}}(M)$ concentrated in degree 0 , and $\left[H_{Q}^{d+1}\left(R_{\mathrm{q}}(M)\right)\right]_{n}=0$ for $n \geqq 0$.

Proof. For brevity we set $M^{*}=R_{\mathrm{q}}(M)$. Since by Corollary 2.3, $\mathfrak{q} M \cap H_{\mathrm{m}}^{0}(M)=0$, we have 


$$
H_{Q}^{0}\left(M^{*}\right)=\bigoplus_{n=0}^{\infty} \mathfrak{q}^{n} M \cap H_{\mathrm{m}}^{0}(M)=H_{\mathrm{m}}^{0}(M)(0) .
$$

To estimate $H_{Q}^{i}\left(M^{*}\right), i \geqq 1$, we consider the exact sequences

$$
\begin{aligned}
& 0 \longrightarrow \mathfrak{q} M^{*}(-1) \longrightarrow M^{*} \longrightarrow M \longrightarrow 0 \\
& 0 \longrightarrow \mathfrak{q} M^{*} \longrightarrow M^{*} \longrightarrow G_{\mathfrak{q}}(M) \longrightarrow 0
\end{aligned}
$$

From the first sequence we get

$$
\left[H_{Q}^{i}\left(\mathfrak{q} M^{*}\right)\right]_{n} \cong\left[H_{Q}^{i}\left(M^{*}\right)\right]_{n+1}
$$

for $n \neq-1$. Using Theorem 5.4, from the second sequence we get

$$
\left[H_{Q}^{i}\left(\mathfrak{q} M^{*}\right)\right]_{n} \cong\left[H_{Q}^{i}\left(M^{*}\right)\right]_{n}
$$

for $n \neq-i, 1-i$, and the exact sequences

$$
\begin{aligned}
0 & \longrightarrow\left[H_{Q}^{i}\left(\mathfrak{q} M^{*}\right)\right]_{-i} \longrightarrow\left[H_{Q}^{i}\left(M^{*}\right)\right]_{-i} \\
{\left[H_{Q}^{i-1}\left(M^{*}\right)\right]_{1-i} } & \longrightarrow H_{m}^{i-1}(M) \longrightarrow\left[H_{Q}^{i}\left(\mathfrak{q} M^{*}\right)\right]_{1-i} \longrightarrow\left[H_{Q}^{i}\left(M^{*}\right)\right]_{1-i} .
\end{aligned}
$$

Since $H_{Q}^{i}\left(M^{*}\right)$ is an artinian module, $\left[H_{Q}^{i}\left(M^{*}\right)\right]_{n}=0$ for $n$ large enough. Hence, from (1) and (2) we get $\left[H_{Q}^{i}\left(M^{*}\right)\right]_{n}=0$ for $n \geqq 0$. Now let $i=1, \cdots, d-1$. Note that by Theorem 5.4 and Proposition $6.1 H_{Q}^{i}\left(M^{*}\right)$ is of finite length. Then $\left[H_{Q}^{i}\left(M^{*}\right)\right]_{n}=0$ for $n$ small enough. Hence, using (1), (2) and (3) we get $\left[H_{Q}^{i}\left(M^{*}\right)\right]_{n}=0$ for $n \leqq 1-i$. Now, from (1), (2) and (4) we can conclude that

$$
\begin{aligned}
& {\left[H_{Q}^{i}\left(M^{*}\right)\right]_{n}=\left[H_{Q}^{i}\left(q M^{*}\right)\right]_{1-\imath}=H_{m}^{i-1}(M)} \\
& R_{\mathrm{a}}(A)^{+}\left[H_{Q}^{i}\left(M^{*}\right)\right]_{n}=0
\end{aligned}
$$

for $i-2 \geqq n \geqq 1$. Thus, $H_{Q}^{i}\left(M^{*}\right)$ is the direct sum of $H_{\mathrm{m}}^{i-1}(M)(n), i-2$ $n \geqq 1$. The proof of Theorem 6.2 is complete.

Remark 6.3. By the statement of Theorem 6.2 we always have $H_{Q}^{1}\left(R_{\mathrm{q}}(M)\right)=0$, and, if $d \geqq 2, H_{Q}^{2}\left(R_{\mathrm{q}}(M)\right)=0$.

The following consequence of Theorem 6.2 is the content of Schenzel's paper [19] which generalizes similar result of Goto and Shimoda in the theory of Buchsbaum rings [7], [12].

COROLlaRy 6.4. Let $\mathfrak{q}$ be an arbitary parameter ideal of $M$. Then $R_{\mathrm{q}}(M)$ is a $C$ - $M$ module iff the following conditions are satisfied:

(i) $H_{\mathrm{m}}^{i}(M)=0$ for $i \neq 1, d$.

(ii) $q H_{\mathrm{m}}^{1}(M)=0$. 
Proof. $\quad \Leftrightarrow)$ First, we have $H_{\mathrm{m}}^{0}(M)=\left[H_{Q}^{0}\left(R_{\mathrm{q}}(M)\right]_{0}=0\right.$. For $i>0$, we consider the exact sequences

$$
\begin{aligned}
& 0 \longrightarrow \mathfrak{q} R_{\mathrm{q}}(M)(-1) \longrightarrow R_{\mathfrak{q}}(M) \longrightarrow M \longrightarrow 0 \\
& 0 \longrightarrow \mathfrak{q} R_{\mathfrak{q}}(M) \longrightarrow R_{\mathfrak{q}}(M) \longrightarrow G_{\mathrm{q}}(M) \longrightarrow 0 .
\end{aligned}
$$

Since $H_{Q}^{i}\left(R_{q}(M)\right)=0, i=0, \cdots, d$, it is easily seen that

$$
H_{Q}^{i}\left(G_{\mathrm{q}}(M)\right) \cong H_{\mathrm{m}}^{i}(M)(1)
$$

for $i=1, \cdots, d-1$. Thus, by Corollary $3.12, a_{1}^{*}, \cdots, a_{d}^{*}$ is a standard s.o.p. of $G_{q}(M)$. Hence, by Corollary 5.5, $a_{1}, \cdots, a_{d}$ is a standard s.o.p. of $M$, which then implies (ii). Moreover, we can compare the above formula for $H_{Q}^{i}\left(G_{q}(M)\right)$ with the one of Theorem 5.4 and immediately get (i).

$(\Leftarrow)$ By Corollary 3.7, $\mathfrak{q}$ is a standard parameter ideal of $M$. Hence, by Theorem $6.2, R_{\mathrm{q}}(M)$ is a C-M module.

Theorem 6.2 may be also applied to study the Rees modules of the module-version of Buchsbaum rings with maximal embedding dimension.

Proposition 6.5. Suppose that $M$ is a generalized $C$ - $M$ module with $d \geqq 2$ and

$$
l\left(M / \mathfrak{a}^{2} M\right)=e(\mathfrak{a} ; M)+I(M)+d l(M / \mathfrak{a} M) .
$$

Then $R_{a}(M)$ is a generalized $C-M$ module with

$$
\begin{aligned}
& H_{Q}^{0}\left(R_{\mathrm{a}}(M)\right)=H_{\mathrm{m}}^{0}(M) \oplus H_{\mathrm{m}}^{0}(M)(-1) \\
& H_{Q}^{1}\left(R_{\mathrm{a}}(M)\right)=H_{\mathrm{m}}^{1}(M) \\
& H_{Q}^{i}\left(R_{\mathrm{a}}(M)\right)=\underset{i-3 \geqq n \geqq 1}{\oplus_{\mathrm{m}}} H^{1-1}(M)(n)
\end{aligned}
$$

for $2 \leqq i \leqq d$, and $\left[H_{Q}^{d+1}\left(R_{\mathrm{a}}(M)\right)\right]_{n}=0$ for $n \geqq 0$.

Proof. Without restriction we may assume that the residue field $k$ is infinite. Let $a_{1}, \cdots, a_{d}$ be a minimal reduction of $\mathfrak{a}$ relative to $M$ (hence to $a M$ too). Then, by Proposition 4.8 (i),

$$
\mathfrak{q} \mathfrak{a}^{n} M=\mathfrak{a}^{n+1} M
$$

for all $n \geqq 1$. By Proposition 4.8 (ii) and Corollary 2.3, $\mathfrak{q} M \cap H_{\mathrm{m}}^{0}(M)=0$. By Corollary 2.6 (iv) and Proposition 4.8 (iii),

$$
H_{\mathrm{m}}^{0}(M)=0_{M}: a_{d} \subseteq \mathfrak{q}_{d-1} M: a_{d} \subseteq \mathfrak{a} M .
$$


Using these facts, it is easy to see that

$$
H_{Q}^{0}\left(R_{\mathrm{a}}(M)\right)=H_{\mathrm{m}}^{0}(M) \oplus H_{\mathrm{m}}^{0}(M)(-1) .
$$

To estimate $H_{Q}^{i}\left(R_{\mathrm{a}}(M)\right), i \geqq 1$, we first consider the exact sequence

$$
0 \longrightarrow a R_{\mathrm{a}}(M)(-1) \longrightarrow R_{\mathrm{a}}(M) \longrightarrow M \longrightarrow 0 .
$$

Then we have

$$
\left[H_{Q}^{i}\left(R_{\mathrm{a}}(M)\right)\right]_{n}=\left[H_{Q}^{i}\left(\mathfrak{a} R_{\mathrm{a}}(M)\right)\right]_{n-1}
$$

for $n \neq 0$. Note that $\mathfrak{a} R_{\mathfrak{a}}(M)=R_{\mathrm{a}}(\mathfrak{a} M)=R_{\mathfrak{q}}(\mathfrak{a} M)$ and that $a_{1}, \cdots, a_{d}$ is a standard s.o.p. of $a M$ by Corollary 4.9 (ii). Then we can apply Theorem 6.2 to $a M$ and get

$$
H_{Q}^{i}\left(\mathfrak{a} R_{\mathfrak{a}}(M)\right)=\underset{i-2 \geqq n \geqq 1}{\bigoplus} H_{\mathrm{m}}^{i-1}(\mathfrak{a} M)(n)
$$

for $i=1, \cdots, d$, and $\left[H_{Q}^{d+1}\left(\mathfrak{a} R_{\mathrm{a}}(M)\right)\right]_{n}=0$ for $n \geqq 0$. Since $H_{\mathfrak{m}}^{i}(\mathfrak{a} M)=$ $H_{\mathrm{m}}^{i}(M)$ for $i>1$, from the above relations we can easily derive the formulas for $H_{Q}^{i}\left(R_{\mathrm{a}}(M)\right)$ of Proposition 6.5 except the following ones:

$$
\begin{aligned}
& {\left[H_{Q}^{1}\left(R_{\mathrm{a}}(M)\right)\right]_{0}=H_{\mathrm{m}}^{1}(M)} \\
& {\left[H_{Q}^{i}\left(R_{\mathrm{a}}(M)\right)\right]_{0}=0}
\end{aligned}
$$

for $i>1$. But these formulas are easily seen from the zero-graded part of the derived local cohomology sequence of the exact sequence

$$
0 \longrightarrow \mathfrak{a} R_{a}(M) \longrightarrow R_{a}(M) \longrightarrow G_{a}(M) \longrightarrow 0
$$

by using the above formula of $H_{Q}^{i}\left(\mathfrak{a} R_{a}(M)\right)$ and the one of $H_{Q}^{i}\left(G_{a}(M)\right)$ of Proposition 5.11.

Remark 6.6. (1) By the statement of Proposition 6.5 we always have $H_{Q}^{2}\left(R_{\mathrm{a}}(M)\right)=0$ and, if $d \geqq 3, H_{Q}^{3}\left(R_{\mathrm{a}}(M)\right)=0$.

(2) If $d=1$, we get another formula for $H_{Q}^{1}\left(R_{\mathrm{a}}(M)\right)$ than the one of Proposition 6.5. Namely, using the same method as in the proof of Proposition 6.5, we can show that in this case,

$$
\begin{aligned}
& H_{Q}^{0}\left(R_{\mathrm{a}}(M)\right)=H_{\mathrm{m}}^{0}(M) \oplus H_{\mathrm{m}}^{0}(M)(-1) \\
& H_{Q}^{1}\left(R_{\mathrm{a}}(M)\right)=0 \\
& {\left[H_{Q}^{2}\left(R_{\mathrm{a}}(M)\right)\right]_{n}=0 \quad \text { for } n \geqq 0 .}
\end{aligned}
$$

From Proposition 5.6 and Remark 6.6 we immediately get the following interesting consequence: 
Corollary 6.7. Let $M$ and $a$ be as in Proposition 6.5 Then $R_{a}(M)$ is a $C$ - $M$ module iff $H_{\mathrm{m}}^{i}(M)=0$ for $i \neq 2, d$.

\section{REFERENCES}

[1] S. S. Abhyankar, Local rings of high embedding dimension, Amer. J. Math., 89 (1967), 1073-1077.

[2] M. Auslander, D. A. Buchsbaum, Codimension and multiplicity, Ann. of Math., 68 (1958), 625-657.

[ 3 ] M. Brodmann, Endlichkeit von lokalen Kohomologie-Moduln arithmetischer Aufblasungen, Preprint.

[4] M. Brodmann, Kohomologische Eigenschaften von Aufblasungen an lokal vollständigen Durchschnitten, Preprint.

[ 5 ] D. A. Buchsbaum, Complexes in local ring theory, In: Some aspects of ring theory, C.I.M.E., Rome 1965.

[6] N. T. Cuong, P. Schenzel, N. V. Trung, Verallgemeinerte Cohen-Macaulay-Moduln, Math. Nachr., 85 (1978), 57-73.

[ 7 ] S. Goto, On the Cohen-Macaulayfication of certain Buchsbaum rings, Nagoya Math. J., 80 (1980), 107-116.

[8] - Buchsbaum rings of maximal embedding dimension, J. Algebra, 76 (1982), 494-508.

[9] —, On the associated graded rings of parameter ideals in Buchsbaum rings, Preprint.

[10] _ Noetherian local rings with Buchsbaum associated graded rings, J. Algebra, 86 (1984), 336-384.

[11] _ - Blowing-up of Buchsbaum rings, Proceedings, Durham symposium on Commutative Algebra, 140-162, London Math. Soc. Lect. Notes, 72 (1982).

[12] S. Goto, Y. Shimoda, On Rees algebras over Buchsbaum rings, J. Math. Kyoto Univ., 20 (1980), 691-708.

[13] J. Herzog, A. Simis, W. V. Vasconcelos, Approximation complexes of blowing-up rings, J. Algebra, 74 (1982), 466-493.

[14] C. Huneke, On the symmetric and Rees algebra generated by a $d$-sequence, J. Algebra, 62 (1980), 268-275.

[15] - The theory of $d$-sequences and powers of ideals, Adv. in Math., 46 (1982), 249-279.

[16] D. G. Northcott, D. Rees, Reductions of ideals in locai rings, Proc. Cambridge Phil. Soc., 50 (1954), 145-158.

[17] J. Sally, Cohen-Macaulay local rings of maximal embedding dimension, J. Algebra, 56 (1979), 168-183.

[18] P. Schenzel, Multiplizitäten in verallgemeinerten Cohen-Macaulay-Moduln, Math. Nachr., 88 (1979), 295-306.

[19] - Regular sequences in Rees and symmetric algebras, Manuscripta Math., 35 (1981), 173-193.

[20] J. Stückrad, W. Vogel, Theorie der Buchsbaum-Moduln, to appear.

[21] J. Stückrad, Über die kohomologische Charakterisierung von Buchsbaum-Moduln, Math. Nachr., 95 (1980), 265-272.

[22] J. Stückrad, W. Vogel, Eine Verallgemeinerung der Cohen-Macaulay-Ringe und Anwendungen auf ein Problem der Multiplizitätstheorie, J. Math. Kyoto Univ., 13 (1973), 513-528.

[23] — Toward a theory of Buchsbaum singularities, Amer. J. Math., 100 (1978), $727-746$. 
[24] N. V. Trung, Über die Übertragung der Ringeigenschaften zwischen $R$ und $R[u] /(F)$, Math. Nachr., 92 (1979), 215-229.

[25] — Some criteria for Buchsbaum modules, Monatsh. Math., 90 (1980), 331-337.

[26] — On the associated graded ring of a Buchsbaum ring, Math. Nachr., 107 (1982), 209-220.

[27] — Absolutely superficial sequence, Math. Proc. Cambridge Phil. Soc., 93 (1983), $35-47$.

[28] G. Valla, Certain graded algebras are always Cohen-Macaulay, J. Algebra, 42 (1976), 537-548.

[29] — On the symmetric and Rees algebras of an ideal, Manuscripta Math., 30 (1980), 239-255.

[30] W. Vogel, Über eine Vermutung von D. A. Buchsbaum, J. Algebra, 25 (1973), 106-112.

[31] - A non-zero-divisor characterization of Buchsbaum modules, Michigan Math. J., 28 (1981), 147-152.

[32] A. Ooishi, Openness of loci, $p$-excellent rings and modules, Hiroshima Math. J., 10 (1980), 419-436.

Institute of Mathematics

Viên Toán hoc

Box 631, Bò̀ Hô, Hanoi, Vietnam 